\title{
A Receiver Structure for Frequency-Flat Time-Varying Rayleigh Channels and Performance Analysis
}

\author{
Xiaofei Shao, Harry Leib* \\ Department of Electrical and Computer Engineering, McGill University, Montreal, Quebec, Canada \\ Email: xiaofei.shao@mail.mcgill.ca, ${ }^{\star}$ harry.leib@mcgill.ca
}

How to cite this paper: Shao, X. and Leib, H. (2016) A Receiver Structure for Frequency-Flat Time-Varying Rayleigh Channels and Performance Analysis. Int. J. Communications, Network and System Sciences, 9, 387-412.

http://dx.doi.org/10.4236/ijcns.2016.910033

Received: June 28, 2016

Accepted: September 25, 2016

Published: September 28, 2016

Copyright $\odot 2016$ by authors and Scientific Research Publishing Inc. This work is licensed under the Creative Commons Attribution International License (CC BY 4.0).

http://creativecommons.org/licenses/by/4.0/ (c) (i) Open Access

\begin{abstract}
This paper proposes a wavelet based receiver structure for frequency-flat time-varying Rayleigh channels, consisting of a receiver front-end followed by a Maximum A-Posteriori (MAP) detector. Discretization of the received continuous time signal using filter banks is an essential stage in the front-end part, where the Fast Haar Transform (FHT) is used to reduce complexity. Analysis of our receiver over slowfading channels shows that it is optimal for certain modulation schemes. By comparison with literature, it is shown that over such channels our receiver can achieve optimal performance for Time-Orthogonal modulation. Computed and Monte-Carlo simulated performance results over fast time-varying Rayleigh fading channels show that with Minimum Shift Keying (MSK), our receiver using four basis functions (filters) lowers the error floor by more than one order of magnitude with respect to other techniques of comparable complexity. Orthogonal Frequency Shift Keying (FSK) can achieve the same performance as Time-Orthogonal modulation for the slow-fading case, but suffers some degradation over fast-fading channels where it exhibits an error floor. Compared to MSK, however, Orthogonal FSK provides better performance.
\end{abstract}

\section{Keywords}

Receiver Structure, Time-Varying Rayleigh Channels, Filter Banks, Fast Haar Transform

\section{Introduction}

Fueled by the increased interest in mobile communication for fast moving platforms [1] [2], signal detection over fast-fading channels has become an important research area in the last decade [3] [4]. When signal fading is slow, the channel over at least one 
symbol interval can be assumed to be Additive White Gaussian Noise (AWGN), and a matched filter receiver front-end followed by symbol rate sampling provides good performance [5]. However, with fast fading the above matched filter method is suboptimal and more advanced techniques are needed [6] [7].

Several methods of receiver design for fast-fading channels have been proposed [8][14]. Pilot symbol assisted modulation [8] adds known symbols in the transmitted signal, allowing the receiver to estimate the channel in order to establish an amplitude and phase reference for detection. This technique improves performance; however it lowers the effective bit rate, introduces delay, and requires buffer space at the receiver for channel interpolation. In [9] it is demonstrated that with fast fading, using a low-pass rectangular pilot filter produces an error floor, and more judiciously designed pilot filters are needed. In [10], the authors show that processing more than one sample per symbol ensures robust performance in a fast-fading environment when Nyquist pulse shaping is used, at the expense of increased system complexity compared to traditional detection techniques. In line with such concept a receiver structure for a fading channel applying multisampling is derived in [11].

Receivers for fast-fading channels based on filter banks are presented in [12]-[14]. In [12], the authors demonstrate two types of receivers based on single-filter and doublefilter. The single-filter receiver consists of two matched filters derived using a time-selective channel model which approximates the fading process by the first two terms of its Taylor expansion. The double-filter receiver consists of two matched filters and two modified matched filters derived using a time-selective channel model approximating the fading process by truncating the Taylor series to the third term. In [13], the authors use specific basis functions as receiver filters for discretization. It is claimed that, by a moderate increase in complexity compared to a matched filter receiver, the performance is close to optimal except at very high Signal-to-Noise Ratio (SNR). Another method of designing front-end filters is presented in [14], that employs the Karhunen-Loeve (KL) expansion [15] to approximate the autocorrelation function of the fading process by a finite dimensional separable kernel.

In this paper, we present a wavelet based receiver for frequency-flat time-varying Rayleigh channels, consisting of two parts: a front-end stage and a Maximum A-Posteriori (MAP) detector. Discretization of the received continuous time signal is an essential function of the front-end stage, and for this task we employ the framework for discrete representation of continuous time signals from [16] that is well suited for fast-fading channels. Furthermore, the Fast Haar Transform (FHT) algorithm [17] is used to reduce complexity. Performance analysis and Monte-Carlo simulation results are presented for three binary modulation schemes: Time-Orthogonal modulation, Minimum Shift Keying (MSK) and Orthogonal Frequency Shift Keying (FSK).

\section{System Model and Discrete Representation of Signals over Time-Varying Rayleigh Channels}

\subsection{System Model and Framework for Discrete Representation}

In this work, we consider a frequency-flat time-varying Rayleigh fading channel, with 
the complex baseband received signal expressed as [18]

$$
r(t)=g(t) s_{m}(t)+n(t), 0 \leq t \leq T
$$

where $s_{m}(t),(m=0,1, \cdots, M-1)$ is transmitted with a-priori probability $p_{m}, g(t)$ is the fading process and $n(t)$ is additive noise. The processes $g(t)$ and $n(t)$ are zero mean complex Gaussian and mutually independent. We assume that $g(t)$ and $n(t)$ have independent real and imaginary components that are stationary with same autocorrelation function. We also assume that $n(t)$ is white with a single-sided power spectral density (PSD) $N_{0}$. We can express (1) in the form

$$
r(t)=g(t) \mathbf{s}^{\mathrm{T}}(t) \mathbf{a}+n(t), 0 \leq t \leq T
$$

where $\mathbf{s}^{\mathrm{T}}(t)=\left[s_{0}(t), s_{1}(t), \cdots, s_{M-1}(t)\right]$, a is a random $M$-dimensional vector with a-priori probability $\operatorname{Pr}\left[\mathbf{a}=\mathbf{1}_{m}\right]=p_{m}$, and $\mathbf{1}_{m}=[0,0, \cdots, 1,0,0]^{\mathrm{T}}$ having 1 as the $m$ th component with the others being 0. Essentially, the vector a selects the signal that is transmitted, and it is independent of $g(t)$ and $n(t)$.

The process of discretization yields a finite dimensional vector of observables from a segment of a continuous time signal. We use the framework of [16] that is based on the KL expansion [15]. We start with the discretization of the message process $y(t)=g(t) \mathbf{s}^{\mathrm{T}}(t) \mathbf{a}$ of mean

$$
E[y(t)]=E\left[g(t) \mathbf{s}^{\mathrm{T}}(t) \mathbf{a}\right]=E[g(t)] \mathbf{s}^{\mathrm{T}}(t) E[\mathbf{a}]=0
$$

since $E[g(t)]=0$, autocorrelation

$$
R_{y}\left(t_{1}, t_{2}\right)=E\left[y\left(t_{1}\right) y^{*}\left(t_{2}\right)\right]=E_{g}\left[E_{a}\left[y\left(t_{1}\right) y^{*}\left(t_{2}\right)\right]\right]=R_{g}\left(t_{1}, t_{2}\right) \sum_{m=0}^{M-1} p_{m} s_{m}\left(t_{1}\right) s_{m}^{*}\left(t_{2}\right)
$$

because

$E_{\mathbf{a}}\left[y\left(t_{1}\right) y^{*}\left(t_{2}\right)\right]=g\left(t_{1}\right) \mathbf{s}^{\mathrm{T}}\left(t_{1}\right) E_{\mathbf{a}}\left[\mathbf{a a}^{\mathrm{T}}\right] \mathbf{s}^{*}\left(t_{2}\right) g^{*}\left(t_{2}\right)=g\left(t_{1}\right) g^{*}\left(t_{2}\right) \sum_{m=0}^{M-1} p_{m} s_{m}\left(t_{1}\right) s_{m}^{*}\left(t_{2}\right)$

and $R_{g}\left(t_{1}, t_{2}\right)=E\left[g\left(t_{1}\right) g^{*}\left(t_{2}\right)\right]$. The KL expansion for $y(t)$ is

$$
y(t)=\sum_{k=1}^{\infty} y_{k} \varphi_{k}(t)
$$

where $y_{k}$ are uncorrelated complex Gaussian variables, and the basis functions $\varphi_{k}(t)$ are obtained by solving the integral equation

$$
\int_{0}^{T} R_{y}\left(t_{1}, t_{2}\right) \varphi_{k}\left(t_{2}\right) \mathrm{d} t_{2}=\lambda_{k} \varphi_{k}\left(t_{1}\right), 0 \leq t_{1} \leq T, 0 \leq t_{2} \leq T .
$$

In (6) we have

$$
y_{k}=\int_{0}^{T} y(t) \varphi_{k}^{*}(t) \mathrm{d} t=\left[y_{k, 0}^{\prime}, \cdots, y_{k, M-1}^{\prime}\right] \mathbf{a}=\mathbf{y}_{k}^{\mathrm{T}} \mathbf{a}
$$

where

$$
y_{k, m}^{\prime}=\int_{0}^{T} g(t) s_{m}(t) \varphi_{k}^{*}(t) \mathrm{d} t .
$$

From the properties of the KL representation, we have

$$
E\left[y_{k} y_{l}^{*}\right]=\lambda_{k} \delta_{k l}
$$


where $\delta_{k l}$ is the Kronecker delta function, and

$$
E\left[y_{k} y_{l}^{*}\right]=E\left[\mathbf{y}_{k}^{\mathrm{T}} \mathbf{a a}^{\mathrm{T}} \mathbf{y}_{l}^{*}\right]=E_{\mathbf{y}}\left[E_{\mathbf{a}}\left[\mathbf{y}_{k}^{\mathrm{T}} \mathbf{a}^{\mathrm{T}} \mathbf{y}_{l}^{*}\right]\right]=\sum_{m=0}^{M-1} p_{m} E_{y}\left[y_{k, m}^{\prime} y_{l, m}^{\prime *}\right] .
$$

\subsection{Examples for Specific Cases}

Slow-Fading Channel with Linear Combination of Orthogonal Signals

The fading process $g(t)=g$ where $g$ has zero mean and autocorrelation $R_{g}\left(t_{1}, t_{2}\right)=E\left[|g|^{2}\right]=\rho_{g}^{2}$, and $s_{m}(t)$ is expressed as

$$
s_{m}(t)=\sum_{p=0}^{P-1} c_{m, p} u_{p}(t)
$$

where $c_{m, p}$ are complex scalars and $u_{p}(t)$ are orthogonal real functions such that

$$
\int_{0}^{T} u_{p}(t) u_{q}(t) \mathrm{d} t=E_{p} \delta_{p q}
$$

with $E_{p}$ denoting the energy of $u_{p}(t)$. In this case, (4) can be written as

$$
R_{y}\left(t_{1}, t_{2}\right)=\rho_{g}^{2} \sum_{m=0}^{M-1} p_{m} \sum_{p=0}^{P-1} c_{m, p} u_{p}\left(t_{1}\right) \sum_{i=0}^{P-1} c_{m, i}^{*} u_{i}\left(t_{2}\right)=\rho_{g}^{2} \sum_{p=0}^{P-1} \sum_{i=0}^{P-1} \beta_{p, i} u_{p}\left(t_{1}\right) u_{i}\left(t_{2}\right)
$$

where $\beta_{p, i}=\sum_{m=0}^{M-1} p_{m} c_{m, p} c_{m, i}^{*}$. After substituting (14) into (7), we have

$$
\lambda_{k} \varphi_{k}\left(t_{1}\right)=\rho_{g}^{2} \sum_{p=0}^{P-1} \sum_{i=0}^{P-1} \beta_{p, i} u_{p}\left(t_{1}\right) \phi_{i}^{(k)}
$$

where $\phi_{i}^{(k)}=\int_{0}^{T} u_{i}\left(t_{2}\right) \varphi_{k}\left(t_{2}\right) \mathrm{d} t_{2}$, showing that the basis functions $\varphi_{k}(t)$ are linear combinations of $\left\{u_{0}(t), \cdots, u_{P-1}(t)\right\}$. The variables $\left\{\phi_{0}^{(k)}, \cdots, \phi_{P-1}^{(k)}\right\}$ and $\lambda_{k}$ can be found by solving a matrix eigen-problem.

Multiplying both sides of (15) by $u_{q}\left(t_{1}\right)$ and integrating results in

$$
\frac{\lambda_{k}}{\rho_{g}^{2}} \phi_{q}^{(k)}=\sum_{i=0}^{P-1} E_{q} \beta_{q, i} \phi_{i}^{(k)}, q=0,1, \cdots, P-1
$$

because of (13). In matrix form, (16) becomes

$$
\lambda_{k}^{\prime} \Phi^{(k)}=\mathbf{G} \Phi^{(k)}
$$

where $\lambda_{k}^{\prime}=\frac{\lambda_{k}}{\rho_{g}^{2}}, \quad \mathbf{G}=\mathbf{E}_{n} \mathbf{B}_{t}, \quad \Phi^{(k)}=\left[\phi_{0}^{(k)}, \phi_{1}^{(k)}, \cdots, \phi_{P-1}^{(k)}\right]^{\mathrm{T}}, \quad \mathbf{E}_{n}=\operatorname{diag}\left(E_{0}, E_{1}, \cdots, E_{P-1}\right)$ with $\operatorname{diag}(\cdot)$ being a block diagonal matrix, and

$$
\mathbf{B}_{t}=\left[\beta_{q, i}\right]_{P \times P} .
$$

We see that (17) is a matrix eigen-problem that can be solved by a multitude of methods.

Orthogonal signaling is a particular case where $c_{m, p}=\delta_{m p}$ and hence

$$
s_{m}(t)=\sum_{p=0}^{P-1} \delta_{m p} u_{p}(t)=u_{m}(t) .
$$

Therefore 


$$
\beta_{p, i}=\sum_{m=0}^{M-1} p_{m} \delta_{m p} \delta_{m i}=p_{p} \delta_{p i},
$$

and the matrix $\mathbf{G}$ in (17) is $\operatorname{diag}\left(E_{0} p_{0}, E_{1} p_{1}, \cdots, E_{P-1} p_{P-1}\right)$, showing that

$$
\begin{gathered}
\phi_{q}^{(k)}=\sqrt{E_{k}} \delta_{q k} \\
\lambda_{k}=\rho_{g}^{2} p_{k} E_{k} .
\end{gathered}
$$

Substituting (20), (21) and (22) into (15) and using (19) yields

$$
\varphi_{k}(t)=\frac{\rho_{g}^{2}}{\lambda_{k}} \sum_{p=0}^{P-1} p_{p} u_{p}(t) \phi_{p}^{(k)}=\frac{1}{\sqrt{E_{k}}} s_{k}(t) .
$$

\section{Frequency-Flat Fast-Fading Rayleigh Channel}

Consider a basis functions $\varphi_{k}(t)$ for the frequency-flat fast-fading Rayleigh channel. From (4), we see that the kernel can be of infinite dimension because of the autocorrelation function $R_{g}\left(t_{1}, t_{2}\right)$. When approximating $R_{g}\left(t_{1}, t_{2}\right)$ as a $N$ dimensional separable kernel, (4) becomes

$$
R_{y}\left(t_{1}, t_{2}\right)=\sum_{n=0}^{N-1 M-1} \sum_{m=0} \alpha_{n} f_{n}\left(t_{1}\right) f_{n}\left(t_{2}\right) p_{m} s_{m}\left(t_{1}\right) s_{m}^{*}\left(t_{2}\right)
$$

where the coefficients $\alpha_{n}$ are calculated to yield a good approximation, and $f_{n}(t)$ are suitable real functions. Denoting $\omega_{i}=\alpha_{n} p_{m}, b_{i}(t)=f_{n}(t) s_{m}(t)$ where $i=n M+m$, (24) becomes

$$
R_{y}\left(t_{1}, t_{2}\right)=\sum_{i=0}^{N M-1} \omega_{i} b_{i}\left(t_{1}\right) b_{i}^{*}\left(t_{2}\right) .
$$

Substituting (25) into (7), we have

$$
\lambda_{k} \varphi_{k}\left(t_{1}\right)=\sum_{i=0}^{N M-1} \omega_{i} b_{i}\left(t_{1}\right) \phi_{i}^{(k)}
$$

where $\phi_{i}^{(k)}=\int_{0}^{T} b_{i}^{*}\left(t_{2}\right) \varphi_{k}\left(t_{2}\right) \mathrm{d} t_{2}$. We see that the basis functions $\varphi_{k}(t)$ are now linear combinations of $\left\{b_{0}(t), \cdots, b_{N M-1}(t)\right\}$. The coefficients of the linear combinations can be formed by solving a matrix eigen-problem. Multiplying both sides of (26) by $b_{j}^{*}\left(t_{1}\right)$ and integrating yields

$$
\lambda_{k} \phi_{j}^{(k)}=\sum_{i=0}^{N M-1} \omega_{i} \gamma_{j, i} \phi_{i}^{(k)}, j=0,1, \cdots, N M-1
$$

where $\gamma_{j, i}=\int_{0}^{T} b_{j}^{*}\left(t_{1}\right) b_{i}\left(t_{1}\right) \mathrm{d} t_{1}$. In matrix form, (27) becomes

$$
\lambda_{k} \Phi^{(k)}=\mathbf{G} \Phi^{(k)}
$$

where $\mathbf{G}=\boldsymbol{\Gamma} \boldsymbol{\Omega}, \boldsymbol{\Phi}^{(k)}=\left[\phi_{0}^{(k)}, \phi_{1}^{(k)}, \cdots, \phi_{N M-1}^{(k)}\right]^{\mathrm{T}}, \quad \Gamma=\left[\gamma_{j, i}\right]_{N M \times N M}$ and $\boldsymbol{\Omega}=\operatorname{diag}\left(\omega_{0}, \omega_{1}, \cdots, \omega_{N M-1}\right)$ We see that $(28)$ is also a matrix eigen-problem. After substituting $\lambda_{k}$ and $\Phi^{(k)}$ into (26), we can compute the basis functions $\varphi_{k}(t)$.

\section{Receiver Structure}

For convenience, we use the normalized time $x=t / T$, expressing the received signal as 


$$
\tilde{r}(x)=\tilde{g}(x) \tilde{s}_{m}(x)+\tilde{n}(x)=\tilde{g}(x) \tilde{\mathbf{s}}^{\mathrm{T}}(x) \mathbf{a}+\tilde{n}(x), 0 \leq x \leq 1
$$

where $\tilde{r}(x)=r(x T), \quad \tilde{g}(x)=g(x T), \quad \tilde{s}_{m}(x)=s_{m}(x T)$ and $\tilde{n}(x)=n(x T)$. The symbol $\sim$ denotes quantities in the normalized time setting. For consistency, $\tilde{n}(x)$ must have a single-sided PSD of $\frac{N_{0}}{T}$. The block diagram of the receiver, illustrated in Figure 1, consists of two parts: a receiver front-end performing the received signal discretization, with output $\tilde{\mathbf{r}}$ used in the second part that is a MAP detector. In Figure 1 FHT stands for Fast Haar Transform [17], and the operator $\operatorname{Vec}(\cdot)$ yields a column vector obtained by concatenating the columns of a matrix.

\subsection{Receiver Front-End}

Operating on $\tilde{r}(x)$, the front-end stage produces the observable vector $\tilde{\mathbf{r}}$ with components

$$
\tilde{r}_{k}=\int_{0}^{1} \tilde{r}(x) \tilde{\varphi}_{k}^{*}(x) \mathrm{d} x .
$$

The basis functions $\tilde{\varphi}_{k}(x)$ can be found using the second example in Section 2.2. In the normalized time setting, the parameters $\alpha_{n}$ and functions $f_{n}(x)$ in (24) are selected using the wavelet-based eigenfunction method in [16], and hence $\alpha_{n}=\mu_{n}$ and $f_{n}(x)=\theta_{n}(x)$ where $\mu_{n}$ and $\theta_{n}(x)$ are eigenvalues and eigenfunctions of the autocorrelation function $R_{\tilde{g}}\left(x_{1}, x_{2}\right)=E\left[\tilde{g}\left(x_{1}\right) \tilde{g}^{*}\left(x_{2}\right)\right]$. Substituting (26) using the normalized time setting into (30), we have

$$
\tilde{r}_{k}=\frac{1}{\tilde{\lambda}_{k}} \sum_{i=0}^{N M-1} \omega_{i} \tilde{\phi}_{i}^{(k) *} \int_{0}^{1} \tilde{r}(x) \tilde{b}_{i}^{*}(x) \mathrm{d} x=\frac{1}{\tilde{\lambda}_{k}} \sum_{i=0}^{N M-1} \omega_{i} \tilde{\phi}_{i}^{(k) *} \xi_{i}
$$
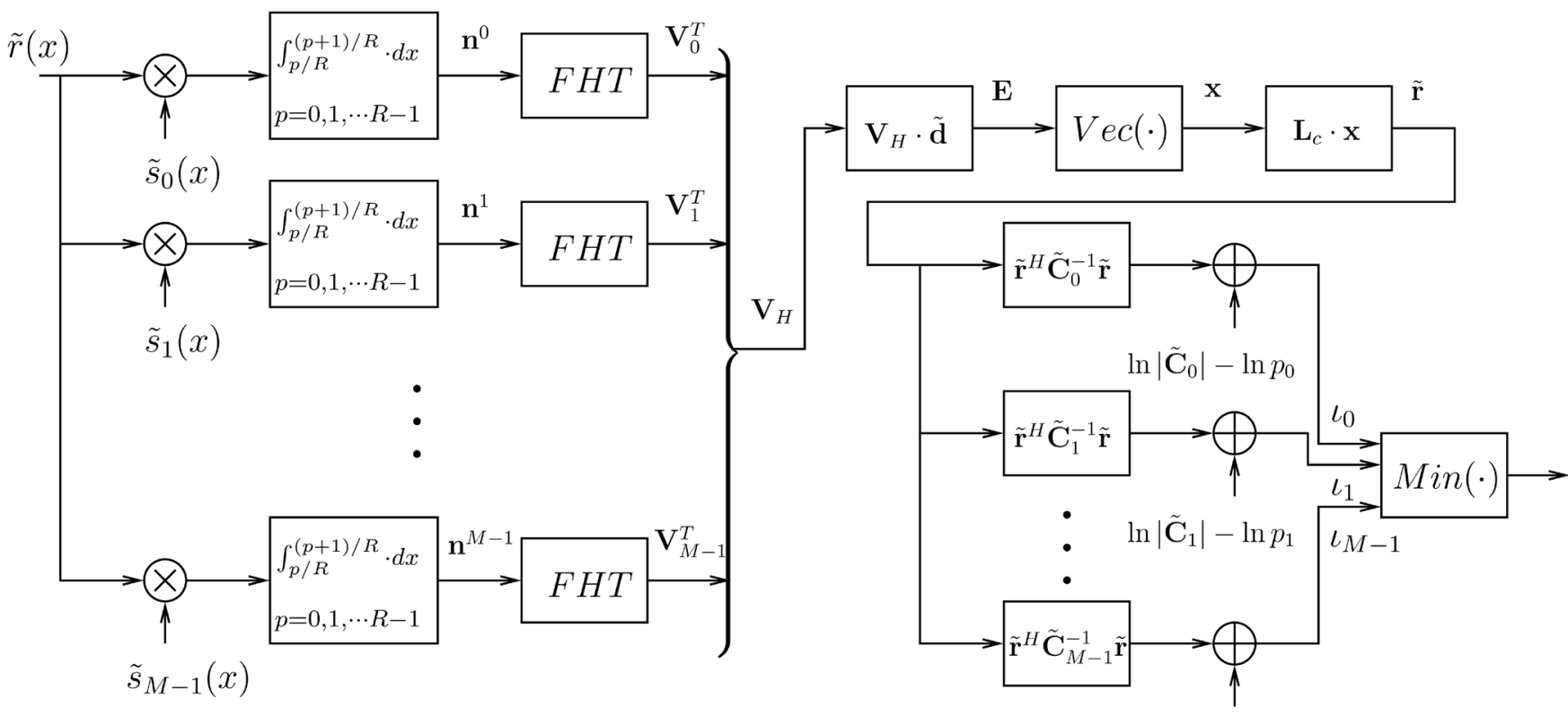

$\ln \left|\tilde{\mathbf{C}}_{M-1}\right|-\ln p_{M-1}$

Figure 1. Receiver block diagram. 
where $\tilde{\phi}_{i}^{(k)}=\int_{0}^{1} \tilde{b}_{i}^{*}(x) \tilde{\varphi}_{k}(x) \mathrm{d} x$ and $\xi_{i}=\int_{0}^{1} \tilde{r}(x) \tilde{b}_{i}^{*}(x) \mathrm{d} x$. In matrix form, (31) can be written as

$$
\tilde{\mathbf{r}}=\mathbf{L}_{c} \cdot \mathbf{x}
$$

where $\mathbf{L}_{c}=\left[l_{k, i}=\tilde{\lambda}_{k}^{-1} \omega_{i} \tilde{\phi}_{i}^{(k) *}\right]_{K \times N M}$ and $\mathbf{x}=\left[\xi_{0}, \xi_{1}, \cdots, \xi_{N M-1}\right]^{\mathrm{T}}$.

Using $\tilde{b}_{i}(x)=\theta_{n}(x) \tilde{s}_{m}(x)$ with $i=n M+m$ and

$$
\theta_{n}(x)=\sum_{l=0}^{L-1} \tilde{d}_{l}^{(n)} \tilde{\psi}_{l}(x),
$$

where $L=2^{c}$ with $c$ the maximum wavelet level, $\tilde{\psi}_{l}(x)$ denoting a family of normalized Haar wavelets including the scaling function with corresponding coefficients $\tilde{d}_{l}^{(n)}$ that can be found using the method from [16], we have

$$
\begin{aligned}
\xi_{i} & =\int_{0}^{1} \tilde{r}(x) \tilde{s}_{m}^{*}(x) \theta_{n}(x) \mathrm{d} x=\int_{0}^{1} \tilde{r}(x) \tilde{s}_{m}^{*}(x) \sum_{l=0}^{L-1} \tilde{d}_{l}^{(n)} \tilde{\psi}_{l}(x) \mathrm{d} x \\
& =e_{m, n}, \quad m=0,1, \cdots, M-1, n=0,1, \cdots, N-1 .
\end{aligned}
$$

Defining $\mathbf{E}=\left[e_{m, n}\right]_{M \times N}$,

$$
\tilde{\mathbf{d}}=\left[\begin{array}{llll}
\tilde{\mathbf{d}}^{(0)} & \tilde{\mathbf{d}}^{(1)} & \ldots & \tilde{\mathbf{d}}^{(N-1)}
\end{array}\right]
$$

with $\tilde{\mathbf{d}}^{(n)}=\left[\tilde{d}_{0}^{(n)}, \tilde{d}_{1}^{(n)}, \cdots, \tilde{d}_{L-1}^{(n)}\right]^{\mathrm{T}}$, we have

$$
\mathbf{x}=\operatorname{Vec}(\mathbf{E})=\operatorname{Vec}\left(\mathbf{V}_{H} \cdot \tilde{\mathbf{d}}\right)
$$

where

$$
\mathbf{V}_{H}=\left[\begin{array}{llll}
\mathbf{V}_{0} & \mathbf{V}_{1} & \cdots & \mathbf{V}_{M-1}
\end{array}\right]^{\mathrm{T}}
$$

with $\mathbf{V}_{m}=\left[V_{m, 0}, V_{m, 1}, \cdots, V_{m, L-1}\right]^{\mathrm{T}}$, and

$$
V_{m, l}=\int_{0}^{1} \tilde{r}(x) \tilde{s}_{m}^{*}(x) \tilde{\psi}_{l}(x) \mathrm{d} x=h_{l}^{-1 / 2} \int_{0}^{1} \tilde{r}(x) \tilde{s}_{m}^{*}(x) \psi_{l}(x) \mathrm{d} x .
$$

In (38), $h_{l}$ are normalization factors [19], and $\psi_{l}(x)$ are a family of unnormalized Haar wavelets [19] with scaling function

$$
\psi_{0}(x)= \begin{cases}1, & 0 \leq x<1 \\ 0, & \text { else }\end{cases}
$$

and

$$
\psi_{l}(x)=\psi\left(2^{s} x-f\right)
$$

where $s=0,1, \cdots, c-1, \quad f=0,1, \cdots, 2^{s}-1, \quad l=2^{s}+f$, and mother function [19]

$$
\psi(x)= \begin{cases}1, & 0 \leq x<1 / 2 \\ -1, & 1 / 2 \leq x<1 \\ 0, & \text { else }\end{cases}
$$

Next, we define $R \geq L$ as the resolution of $\psi_{l}(x)$, and hence we can divide (38) into $R$ sub-integrals resulting in 


$$
V_{m, l}=h_{l}^{-1 / 2} \sum_{p=0}^{R-1} \psi_{p}^{l} \int_{\frac{p}{R}}^{\frac{p+1}{R}} \tilde{r}(x) \tilde{s}_{m}^{*}(x) \mathrm{d} x,
$$

where $\psi_{p}^{l},(p=0,1, \cdots, R-1)$ are samples of $\psi_{l}(x)$ given by

$$
\psi_{p}^{l}=\psi_{l}\left(\frac{2 p+1}{2 R}\right)
$$

since $\psi_{l}(x)$ are constant over each integration sub-interval. From (42), we have

$$
V_{m, l}=h_{l}^{-1 / 2} \Psi^{l} \mathbf{n}^{m}
$$

where $\Psi^{l}=\left[\psi_{0}^{l}, \psi_{1}^{l}, \cdots, \psi_{R-1}^{l}\right]$ and $\mathbf{n}^{m}=\left[\eta_{0}^{m}, \eta_{1}^{m}, \cdots, \eta_{R-1}^{m}\right]^{\mathrm{T}}$ with

$$
\eta_{p}^{m}=\int_{\frac{p}{R}}^{\frac{p+1}{R}} \tilde{r}(x) \tilde{s}_{m}^{*}(x) \mathrm{d} x .
$$

For conceptual simplicity, we take $R=L$ since a larger $R$ does not affect the value of $V_{m, l}$. Therefore, from (44), we have

$$
\mathbf{V}_{m}=\mathbf{H}^{-1 / 2} \Psi \mathbf{n}^{m}
$$

where $\mathbf{H}=\operatorname{diag}\left(h_{0}, h_{1}, \cdots, h_{L-1}\right)$ and $\Psi=\left[\begin{array}{llll}\Psi^{0^{\mathrm{T}}} & \Psi^{1^{\mathrm{T}}} & \ldots & \Psi^{L-1^{\mathrm{T}}}\end{array}\right]^{\mathrm{T}}$. To solve (46) when $R$ is large, we can use the FHT algorithm that has a computational complexity $O(N)$ where $N$ is the number of input elements [17].

\subsection{MAP Detector}

The observable vector (31) is zero mean jointly Gaussian with conditional Probability Density Function (PDF)

$$
f\left(\tilde{\mathbf{r}} \mid \tilde{S}_{m}(x)\right)=\frac{1}{\pi^{K}\left|\tilde{\mathbf{C}}_{m}\right|} \mathrm{e}^{-\tilde{\mathbf{r}}^{H} \tilde{\mathbf{C}}_{m}^{-1} \tilde{\mathbf{r}}}
$$

where $\tilde{\mathbf{r}}=\left[\tilde{r}_{1}, \tilde{r}_{2}, \cdots, \tilde{r}_{K}\right]^{\mathrm{T}}$ and

$$
\begin{aligned}
\tilde{\mathbf{C}}_{m} & =E\left[\tilde{\mathbf{r}} \cdot \tilde{\mathbf{r}}^{H} \mid \tilde{s}_{m}(x)\right]=E\left[\tilde{\mathbf{y}} \cdot \tilde{\mathbf{y}}^{H} \mid \tilde{s}_{m}(x)\right]+E\left[\tilde{\mathbf{n}} \cdot \tilde{\mathbf{n}}^{H}\right] \\
& =\left[\tilde{c}_{k, l}=E\left[\tilde{y}_{k} \tilde{y}_{l}^{*} \mid \tilde{s}_{m}(x)\right]\right]_{K \times K}+\frac{N_{0}}{T} \mathbf{I}_{K}
\end{aligned}
$$

with $\tilde{\mathbf{y}}=\left[\tilde{y}_{1}, \tilde{y}_{2}, \cdots, \tilde{y}_{K}\right]^{\mathrm{T}}$ where $\tilde{y}_{k}$ is defined in (8) using the normalized time setting, $\tilde{\mathbf{n}}=\left[\tilde{n}_{1}, \tilde{n}_{2}, \cdots, \tilde{n}_{K}\right]^{\mathrm{T}}$, and

$$
\begin{aligned}
E\left[\tilde{y}_{k} \tilde{y}_{l}^{*} \mid \tilde{s}_{m}(x)\right]= & \sum_{i=0}^{N M-1} \frac{\omega_{i} \tilde{\phi}_{i}^{(k) *}}{a_{k} \tilde{\lambda}_{k}} \sum_{j=0}^{N M-1} \frac{\omega_{j} \tilde{\phi}_{j}^{(l)}}{a_{l} \tilde{\lambda}_{l}} \sum_{e=0}^{L-1} \frac{\tilde{d}_{e}^{(n)}}{h_{e}^{1 / 2}} \sum_{f=0}^{L-1} \frac{\tilde{d}_{f}^{(w)}}{h_{f}^{1 / 2}} \sum_{p=0}^{R-1} \psi_{p}^{e} \sum_{q=0}^{R-1} \psi_{q}^{f} \\
& \cdot \int_{\frac{p}{R}}^{\frac{p+1}{R}} \int_{\frac{q}{R}}^{\frac{q+1}{R}} R_{\tilde{g}}\left(x_{1}, x_{2}\right) \tilde{s}_{m}\left(x_{1}\right) \tilde{s}_{u}^{*}\left(x_{1}\right) \tilde{s}_{m}^{*}\left(x_{2}\right) \tilde{s}_{v}\left(x_{2}\right) \mathrm{d} x_{1} \mathrm{~d} x_{2} .
\end{aligned}
$$

The normalization factors ensuring $\tilde{\varphi}_{k}(x)$ have unit energy are derived in the Appendix. In (48), $E\left[\tilde{\mathbf{n}} \cdot \tilde{\mathbf{n}}^{H}\right]$ is obtained by using

$$
E\left[\tilde{n}_{k} \tilde{n}_{l}^{*}\right]=\int_{0}^{1} \int_{0}^{1} E\left[\tilde{n}\left(x_{1}\right) \tilde{n}^{*}\left(x_{2}\right)\right] \tilde{\varphi}_{k}^{*}\left(x_{1}\right) \tilde{\varphi}_{l}\left(x_{2}\right) \mathrm{d} x_{1} \mathrm{~d} x_{2}
$$




$$
=\int_{0}^{1} \int_{0}^{1} \frac{N_{0}}{T} \delta\left(x_{1}-x_{2}\right) \tilde{\varphi}_{k}^{*}\left(x_{1}\right) \tilde{\varphi}_{l}\left(x_{2}\right) \mathrm{d} x_{1} \mathrm{~d} x_{2}=\frac{N_{0}}{T} \delta_{k l} .
$$

The structure of the MAP detector can be simplified by using the log-domain

$$
\ln \left[f\left(\tilde{\mathbf{r}} \mid \tilde{S}_{m}(x)\right) p_{m}\right]=-\left(\tilde{\mathbf{r}}^{H} \tilde{\mathbf{C}}_{m}^{-1} \tilde{\mathbf{r}}+K \ln \pi+\ln \left|\tilde{\mathbf{C}}_{m}\right|-\ln p_{m}\right) .
$$

Since $K \ln \pi$ is constant, finding the maximum value of $f\left(\tilde{\mathbf{r}} \mid \tilde{s}_{m}(x)\right) p_{m}$ is equivalent to finding the minimum over the $M \log$-likelihood metrics

$$
\iota_{m}=\tilde{\mathbf{r}}^{H} \tilde{\mathbf{C}}_{m}^{-1} \tilde{\mathbf{r}}+\ln \left|\tilde{\mathbf{C}}_{m}\right|-\ln p_{m} .
$$

\subsection{Structural Analysis of the Receiver over Slow-Fading Channels}

In this case the fading process satisfies $\tilde{g}(x)=g$ where $E[g]=0$, $R_{\tilde{g}}\left(x_{1}, x_{2}\right)=E\left[|g|^{2}\right]=\rho_{g}^{2}$ with eigenvalues [16]

$$
\mu_{n}=\left\{\begin{array}{ll}
\rho_{g}^{2} & \text { for } n=0 \\
0 & \text { for } n=1,2, \cdots, N-1
\end{array},\right.
$$

and (35) is of the form

$$
\tilde{\mathbf{d}}=\left[\begin{array}{llll}
\tilde{\mathbf{d}}^{(0)} & \tilde{\mathbf{d}}^{(1)} & \cdots & \tilde{\mathbf{d}}^{(N-1)}
\end{array}\right]=\left[\begin{array}{cccc}
1 & 0 & \cdots & 0 \\
0 & 0 & \cdots & 0 \\
\vdots & \vdots & \ddots & \vdots \\
0 & 0 & \cdots & 0
\end{array}\right]_{L \times N} .
$$

Hence, (36) becomes

$$
\mathbf{x}=\left[V_{0,0}, V_{1,0}, \cdots, V_{M-1,0}, 0, \cdots, 0\right]^{\mathrm{T}} .
$$

Because $\omega_{i}=\mu_{n} p_{m}$ with $i=n M+m$, from (54) we have

$$
\omega_{i}= \begin{cases}\rho_{g}^{2} p_{i} & \text { for } i=0,1, \cdots, M-1 \\ 0 & \text { for } i=M, M+1, \cdots, N M-1\end{cases}
$$

and (32) becomes

$$
\begin{aligned}
& \tilde{\mathbf{r}}=\left[\tilde{r}_{1}, \tilde{r}_{2}, \cdots, \tilde{r}_{K}\right]^{\mathrm{T}} \\
& =\left[\begin{array}{ccccccc}
\tilde{\lambda}_{1}^{-1} \rho_{g}^{2} p_{0} \tilde{\phi}_{0}^{(1) *} & \tilde{\lambda}_{1}^{-1} \rho_{g}^{2} p_{1} \tilde{\phi}_{1}^{(1) *} & \cdots & \tilde{\lambda}_{1}^{-1} \rho_{g}^{2} p_{M-1} \tilde{\phi}_{M-1}^{(1) *} & 0 & \cdots & 0 \\
\tilde{\lambda}_{2}^{-1} \rho_{g}^{2} p_{0} \tilde{\phi}_{0}^{(2) *} & \tilde{\lambda}_{2}^{-1} \rho_{g}^{2} p_{1} \tilde{\phi}_{1}^{(2) *} & \cdots & \tilde{\lambda}_{2}^{-1} \rho_{g}^{2} p_{M-1} \tilde{\phi}_{M-1}^{(2) *} & 0 & \cdots & 0 \\
\vdots & \vdots & \ddots & \vdots & \vdots & \ddots & \vdots \\
\tilde{\lambda}_{K}^{-1} \rho_{g}^{2} p_{0} \tilde{\phi}_{0}^{(K) *} & \tilde{\lambda}_{K}^{-1} \rho_{g}^{2} p_{1} \tilde{\phi}_{1}^{(K) *} & \cdots & \tilde{\lambda}_{K}^{-1} \rho_{g}^{2} p_{M-1} \tilde{\phi}_{M-1}^{(K) *} & 0 & \cdots & 0
\end{array}\right]_{K \times N M} \\
& \cdot\left[V_{0,0}, V_{1,0}, \cdots, V_{M-1,0}, 0, \cdots, 0\right]^{\mathrm{T}} .
\end{aligned}
$$

Therefore, we have $\tilde{r}_{k}=\sum_{i=0}^{M-1} \tilde{\lambda}_{k}^{-1} \rho_{g}^{2} p_{i} \tilde{\phi}_{i}^{(k) *} V_{i, 0}$, showing that $\tilde{r}_{k}$ are linear combinations of $p_{i} \tilde{\phi}_{i}^{(k) *} V_{i, 0}$. From (42) we have

$$
V_{i, 0}=\sum_{p=0}^{R-1} \int_{\frac{p}{R}}^{\frac{p+1}{R}} \tilde{r}(x) \tilde{s}_{i}^{*}(x) \mathrm{d} x=\int_{0}^{1} \tilde{r}(x) \tilde{s}_{i}^{*}(x) \mathrm{d} x
$$


since $h_{0}=1$ and $\psi_{p}^{0}=1$ for $p=0,1, \cdots, R-1$. We can simplify (57) by removing the zeros, and assuming $p_{i}=\frac{1}{M}$. Then, with $\mathbf{x}^{\prime}=\left[V_{0,0}, V_{1,0}, \cdots, V_{M-1,0}\right]^{\mathrm{T}}$ and

$$
\mathbf{L}_{c}^{\prime}=\left[l_{k, i}^{\prime}=\tilde{\lambda}_{k}^{-1} \rho_{g}^{2} M^{-1} \tilde{\phi}_{i}^{(k) *}\right]_{K \times M}
$$

we have

$$
\tilde{\mathbf{r}}=\mathbf{L}_{c} \cdot \mathbf{x}=\mathbf{L}_{c}^{\prime} \cdot \mathbf{x}^{\prime} .
$$

Furthermore, since $R_{\tilde{g}}\left(x_{1}, x_{2}\right)=\rho_{g}^{2}$, (4) in the normalized time setting can be expressed as

$$
R_{\tilde{y}}\left(x_{1}, x_{2}\right)=\rho_{g}^{2} \sum_{m=0}^{M-1} p_{m} \tilde{s}_{m}\left(x_{1}\right) \tilde{s}_{m}^{*}\left(x_{2}\right),
$$

and substituting this into (7) yields

$$
\begin{aligned}
& \rho_{g}^{2} \sum_{m=0}^{M-1} p_{m} \tilde{s}_{m}\left(x_{1}\right) \int_{0}^{1} \tilde{s}_{m}^{*}\left(x_{2}\right) \tilde{\varphi}_{k}\left(x_{2}\right) \mathrm{d} x_{2}=\tilde{\lambda}_{k} \tilde{\varphi}_{k}\left(x_{1}\right) \\
& \rho_{g}^{2} \sum_{m=0}^{M-1} p_{m} \tilde{s}_{m}\left(x_{1}\right) \tilde{\phi}_{m}^{(k)}=\tilde{\lambda}_{k} \tilde{\varphi}_{k}\left(x_{1}\right)
\end{aligned}
$$

where

$$
\tilde{\phi}_{m}^{(k)}=\int_{0}^{1} \tilde{s}_{m}^{*}(x) \tilde{\varphi}_{k}(x) \mathrm{d} x .
$$

In ([20], p. 170], the authors present optimum receivers for slow-fading channels. From Figure 2, it is seen that in order to prove that our receiver can achieve optimality, we need to focus on two components: the quadratic form $\tilde{\mathbf{r}}^{H} \tilde{\mathbf{C}}_{m}^{-1} \tilde{\mathbf{r}}$ and the bias term $\ln \left|\tilde{\mathbf{C}}_{m}\right|$, since the major differences between our receiver and the optimum receiver from ([20], p. 170) are in these components. Using (61), the quadratic form in Figure 2 can be written as

$$
\tilde{\mathbf{r}}^{H} \tilde{\mathbf{C}}_{m}^{-1} \tilde{\mathbf{r}}=\mathbf{x}^{\prime H} \mathbf{L}_{c}^{\prime H} \tilde{\mathbf{C}}_{m}^{-1} \mathbf{L}_{c}^{\prime} \mathbf{x}^{\prime} .
$$

Compared to ([20], p. 170], our receiver needs to satisfy the following two conditions to achieve optimality:

Condition 1

$$
\ln \left|\tilde{\mathbf{C}}_{m}\right|=\ln \left(1+S N R_{m}\right)+C_{B}
$$

where $C_{B}$ is a constant and $S N R_{m}$ is defined as

$$
S N R_{m}=\frac{E\left[|g|^{2}\right] \cdot E_{m}}{N_{0}}=\frac{\rho_{g}^{2} \int_{0}^{T}\left|S_{m}(t)\right|^{2} \mathrm{~d} t}{N_{0}}=\frac{\rho_{g}^{2} \int_{0}^{1}\left|\tilde{S}_{m}(x)\right|^{2} \mathrm{~d} x}{N_{0} / T}=\frac{\rho_{g}^{2} \tilde{E}_{m}}{N_{0} / T} .
$$

Condition 2

$$
\mathbf{L}_{c}^{\prime H} \tilde{\mathbf{C}}_{m}^{-1} \mathbf{L}_{c}^{\prime}=\mathbf{B}_{m}+\mathbf{M}_{c}
$$

where $\mathbf{M}_{c}$ is a $M \times M$ constant matrix, $\mathbf{B}_{m}$ has the form 

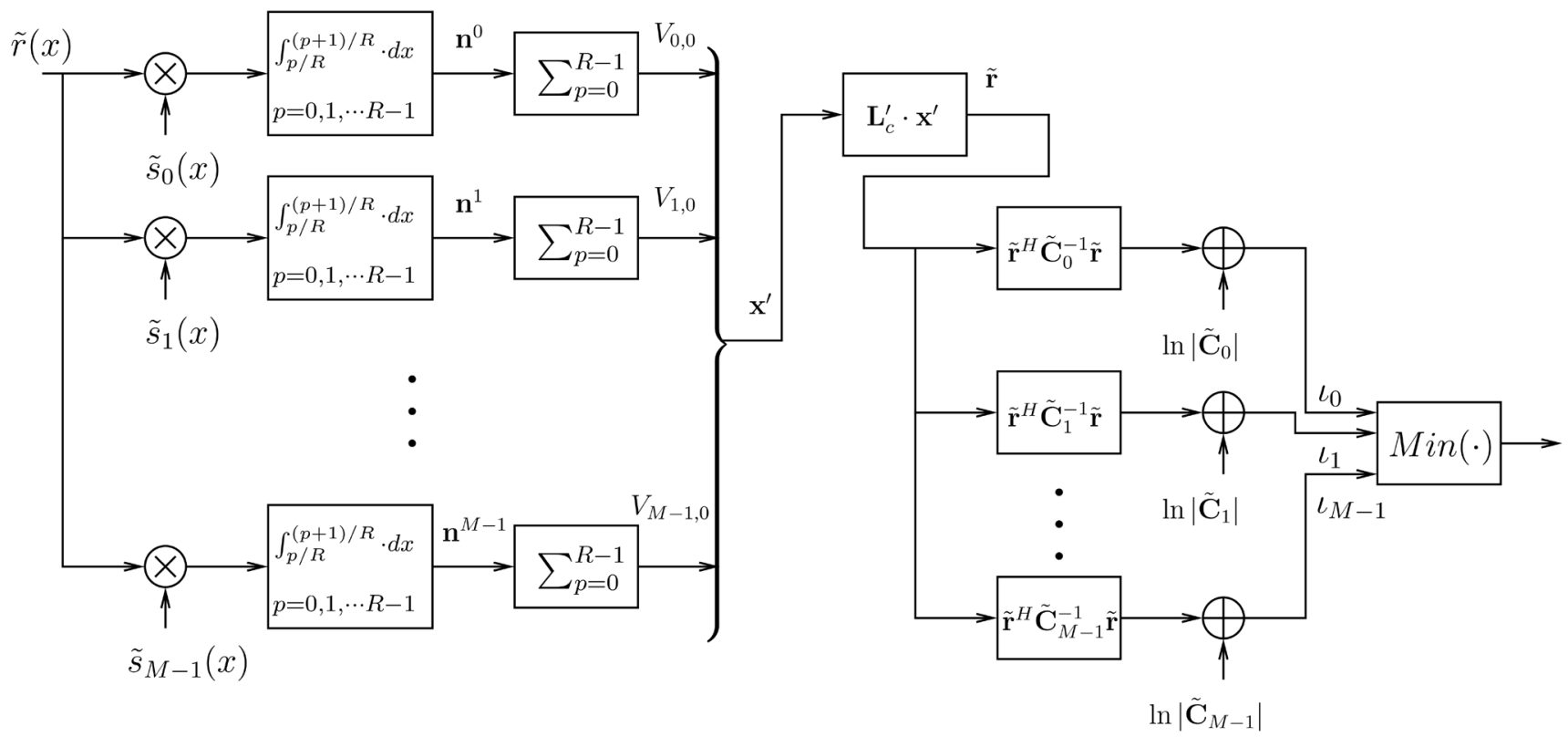

Figure 2. Simplified receiver block diagram.

$$
\mathbf{B}_{m}=\left[\begin{array}{cccc}
0 & 0 & \cdots & 0 \\
0 & b_{m, m} & \cdots & 0 \\
\vdots & \vdots & \ddots & \vdots \\
0 & 0 & \cdots & 0
\end{array}\right]
$$

and

$$
b_{m, m}=\frac{c_{S}}{E_{m} N_{0}} \cdot \frac{S N R_{m}}{1+S N R_{m}}
$$

with $c_{S}$ a constant.

From section B of the Appendix we have that satisfying

$$
\tilde{\phi}_{m}^{(k)}=\int_{0}^{1} \tilde{S}_{m}^{*}(x) \tilde{\varphi}_{k}(x) \mathrm{d} x=\sqrt{\tilde{E}_{m}} \delta_{m k}
$$

where $\frac{\tilde{E}_{m}}{\tilde{\lambda}_{m}}$ is a constant sufficient for Conditions 1 and 2 to hold. Assume that the transmitted signals $\tilde{s}_{m}(x)$ are orthogonal

$$
\int_{0}^{1} \tilde{S}_{m}(x) \tilde{S}_{n}^{*}(x) \mathrm{d} x=\tilde{E}_{m} \delta_{m n},
$$

and equiprobable $p_{m}=\frac{1}{M}$. From the first example of Section 2.2 when applied to orthogonal signaling in the normalized time setting, we have

$$
\begin{aligned}
& \tilde{\lambda}_{k}=\rho_{g}^{2} \tilde{E}_{k} / M, \\
& \tilde{\phi}_{m}^{(k)}=\int_{0}^{1} \tilde{S}_{m}^{*}(x) \tilde{\phi}_{k}(x) \mathrm{d} x=\sqrt{\tilde{E}_{k}} \delta_{m k} .
\end{aligned}
$$

From (73), it is seen that $\frac{\tilde{E}_{k}}{\tilde{\lambda}_{k}}$ is constant. Because of (73) and (74), both Conditions 
1 and 2 hold, showing that our receiver with orthogonal signaling is optimal for slowfading channels. Next we consider the performance over fast-fading channels.

\section{Performance Analysis for Binary Modulation}

\subsection{Error Probability}

From (53), using the log-likelihood metrics for hypotheses $H_{0}\left(\tilde{s}_{0}(t)\right.$ was transmitted) and $H_{1}\left(\tilde{s}_{1}(t)\right.$ was transmitted), the log-likelihood ratio can be expressed as

$$
\Lambda_{0,1}=\iota_{1}-\imath_{0}=\tilde{\mathbf{r}}^{H}\left(\tilde{\mathbf{C}}_{1}^{-1}-\tilde{\mathbf{C}}_{0}^{-1}\right) \tilde{\mathbf{r}}-\ln \frac{p_{1}\left|\tilde{\mathbf{C}}_{0}\right|}{p_{0}\left|\tilde{\mathbf{C}}_{1}\right|} .
$$

Thus, we have the log-likelihood decision rule

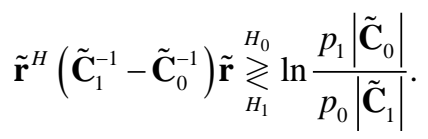

Defining $\tilde{\Lambda}_{0,1}=\tilde{\mathbf{r}}^{H}\left(\tilde{\mathbf{C}}_{1}^{-1}-\tilde{\mathbf{C}}_{0}^{-1}\right) \tilde{\mathbf{r}}$ and the bias term $A_{0,1}=\ln \frac{p_{1}\left|\tilde{\mathbf{C}}_{0}\right|}{p_{0}\left|\tilde{\mathbf{C}}_{1}\right|}$, then $\tilde{\Lambda}_{0,1}$ is a Hermitian quadratic form where $\tilde{\mathbf{C}}_{1}^{-1}-\tilde{\mathbf{C}}_{0}^{-1}$ is a Hermitian matrix because $\tilde{\mathbf{C}}_{0}^{-1}$ and $\tilde{\mathbf{C}}_{1}^{-1}$ are Hermitian. The observable vector $\tilde{\mathbf{r}}$ is zero mean jointly Gaussian, and the conditional PDF of $\tilde{\mathbf{r}}$ is given in (47). The characteristic function of the Hermitian quadratic form $\tilde{\Lambda}_{0,1}$ is given by [21]

$$
\Delta_{\tilde{\Lambda}_{0,1}}(j \omega)=\left|\mathbf{I}_{K}-j \omega \tilde{\mathbf{C}}_{0}\left(\tilde{\mathbf{C}}_{1}^{-1}-\tilde{\mathbf{C}}_{0}^{-1}\right)\right|^{-1}=\left|\mathbf{I}_{K}-j \omega \mathbf{R}_{0,1}\right|^{-1}=\prod_{i=1}^{K}\left(1-j \omega \mu_{i}^{0,1}\right)^{-1}
$$

where $\mathbf{R}_{0,1}=\tilde{\mathbf{C}}_{0}\left(\tilde{\mathbf{C}}_{1}^{-1}-\tilde{\mathbf{C}}_{0}^{-1}\right)$ and $\mu_{i}^{0,1}$ are the eigenvalues of $\mathbf{R}_{0,1}$. Assuming $\tilde{s}_{0}(x)$ is transmitted but $\tilde{s}_{1}(x)$ is detected and denoting $z=j \omega$, the Pairwise Error Probability (PEP) is [18]

$$
\begin{aligned}
P_{p}^{0,1} & =\operatorname{Pr}\left[\tilde{s}_{0}(x) \rightarrow \tilde{s}_{1}(x) \mid \tilde{s}_{0}(x)\right]=\operatorname{Pr}\left[\tilde{\Lambda}_{0,1}<A_{0,1} \mid \tilde{s}_{0}(x)\right] \\
& =\frac{1}{2 \pi j} \int_{-\infty}^{A_{0,1}} \int_{-j \infty}^{+j \infty} \Delta_{\tilde{\Lambda}_{0,1}}(z) \mathrm{e}^{-z u} \mathrm{~d} z \mathrm{~d} u
\end{aligned}
$$

where $\frac{1}{2 \pi j} \int_{-j \infty}^{+j \infty} \Delta_{\tilde{\Lambda}_{0,1}}(z) \mathrm{e}^{-z u} \mathrm{~d} z$ is the PDF of $\tilde{\Lambda}_{0,1}$. Using similar methods as in [22] aided by the residue theorem [23], we have from (78)

$$
P_{p}^{0,1}=\sum_{\substack{i, \mu_{i}^{0,1}<0}}^{K} \prod_{j \neq i}^{K}\left(1-\mu_{j}^{0,1} / \mu_{i}^{0,1}\right)^{-1} \mathrm{e}^{-A_{0,1} / \mu_{i}^{0,1}}
$$

for $A_{0,1} \leq 0$, and

$$
P_{p}^{0,1}=1-\sum_{\substack{i \\ \mu_{i}^{0,1}>0}} \prod_{j \neq i}^{K}\left(1-\mu_{j}^{0,1} / \mu_{i}^{0,1}\right)^{-1} \mathrm{e}^{-\mathrm{A}_{0,1} / \mu_{i}^{0,1}}
$$

for $A_{0,1}>0$.

In our work the PEP was calculated from (79) (80) using the MATLAB software 
package. We consider two fading autocorrelation functions: the Jakes' model [24]

$$
R_{\tilde{g}}\left(x_{1}, x_{2}\right)=E\left[\tilde{g}\left(x_{1}\right) \tilde{g}^{*}\left(x_{2}\right)\right]=J_{0}\left(2 \pi f_{d T}\left|x_{1}-x_{2}\right|\right),
$$

and autocorrelation function of a Butterworth filtered fading process [12]

$$
R_{\tilde{g}}\left(x_{1}, x_{2}\right)=\frac{1}{2} \exp \left(-2 \pi f_{d T}\left|x_{1}-x_{2}\right|\right)+\exp \left(-\pi f_{d T}\left|x_{1}-x_{2}\right|\right) \sin \left(\frac{\pi}{6}+\sqrt{3} \pi f_{d T}\left|x_{1}-x_{2}\right|\right)
$$

where $f_{d T}=f_{d} T$ is the normalized Doppler spread. We assume that $\tilde{s}_{m}(x)$ are equipropable $p_{0}=p_{1}=\frac{1}{2}$. From (79) and (80), it is seen that the error performance is determined by $\mu_{i}^{0,1}$ which are the eigenvalues of $\mathbf{R}_{0,1}=\tilde{\mathbf{C}}_{0}\left(\tilde{\mathbf{C}}_{1}^{-1}-\tilde{\mathbf{C}}_{0}^{-1}\right)$ and $A_{0,1}=\ln \frac{\left|\tilde{\mathbf{C}}_{0}\right|}{\left|\tilde{\mathbf{C}}_{1}\right|}$. The covariance matrix $\tilde{\mathbf{C}}_{m}$ is given by (48) with its components obtained from (49). The double integrations in (49) are computed numerically using the MATLAB function quad $2 d$ with an absolute accuracy of $10^{-23}$. The eigenvalues $\mu_{i}^{0,1}$ are computed using the function eig which calculates eigenvalues of a symbolic matrix and ensures accuracy to at least 32 significant decimal digits by default. The SNR for performance analysis is defined as

$$
S N R=\frac{\bar{E}_{b}}{N_{0}}=\frac{\frac{1}{2} \sum_{m=0}^{1} \int_{0}^{T} E\left[|g(t)|^{2}\right]\left|S_{m}(t)\right|^{2} \mathrm{~d} t}{N_{0}}=\frac{\frac{1}{2} \sum_{m=0}^{1} \int_{0}^{1}\left|\tilde{s}_{m}(x)\right|^{2} \mathrm{~d} x}{N_{0} / T}
$$

where, using (81) and (82), we have $E\left[|g(t)|^{2}\right]=E\left[|\tilde{g}(x)|^{2}\right]=1$. The accuracy of the performance analysis is confirmed by computer simulations.

\subsection{Computer Simulations}

Computer simulations in this paper employ the Monte-Carlo method and are implemented in the $\mathrm{C}$ language. We implemented the receiver of Figure 1 with three binary modulation schemes: Time-Orthogonal modulation, MSK, and Orthogonal FSK. The Bit Error Rate (BER) is estimated from at least 400 errors. In addition, we run at least 10,000 fading channel realizations to ensure accuracy. To emulate continuous time signals we massively oversample by using 4096 samples per symbol interval.

For the Jakes' model, we use the Rayleigh fading channel simulator of [25] that is based on the sum-of-sinusoids algorithm, where we employ 50 sinusoids. Since we oversample, the Jakes' model is expressed as $J_{0}\left(2 \pi f_{d T}\left|\hat{x}_{p}-\hat{x}_{q}\right|\right)=J_{0}\left(2 \pi \frac{f_{d T}}{S}|p-q|\right)$ where $\hat{x}_{p}=\frac{2 p+1}{2 S}, \quad p=0,1, \cdots, S-1$ and $q=0,1, \cdots, S-1$ with $S$ the total number of samples taken per symbol interval. For the Butterworth lowpass filtered fading process, each fading realization is generated by passing two white and independent real Gaussian processes through two identical third-order Butterworth filters as in [12]. The $3 \mathrm{~dB}$ bandwidth of these filters, $f_{d}$, is a measure of the fading rate.

The SNR for simulations can be expressed as $S N R=\frac{\bar{P}_{b}}{P_{n}}=\frac{\bar{E}_{b} / T}{P_{n}}$ where 


$$
\bar{E}_{b}=\frac{1}{2} \sum_{m=0}^{1} E\left[\int_{0}^{T}|g(t)|^{2}\left|S_{m}(t)\right|^{2} \mathrm{~d} t\right]=\frac{T}{2} \sum_{m=0}^{1} \int_{0}^{1}\left|\tilde{s}_{m}(x)\right|^{2} \mathrm{~d} x .
$$

After passing the received signal through an ideal band-limiting anti-aliasing filter, the power spectral density of $n(t)$ becomes

$$
p_{n}(f)= \begin{cases}N_{0} & \text { for }-\frac{B}{2} \leq f \leq \frac{B}{2} \\ 0 & \text { otherwise }\end{cases}
$$

with $B=F_{s}$ which is the sampling frequency. Instead of sampling at rate $F_{s}=\frac{1}{T}$, oversampling by a factor $S$ yields $F_{s}=\frac{S}{T}$. Since the additive noise is zero mean complex Gaussian with independent real and imaginary components which are stationary with same autocorrelation function, the variance of its real (or imaginary) component is given by [26]

$$
\sigma_{c}^{2}=\frac{1}{2} P_{n}=\frac{1}{2} N_{0} B=\frac{1}{2} N_{0} F_{s}=\frac{N_{0}}{2 T} S .
$$

The Time-Orthogonal modulation scheme [13] is defined by the waveforms

$$
\begin{aligned}
& \tilde{s}_{0}(x)= \begin{cases}1 & \text { for } 0 \leq x<1 / 2 \\
0 & \text { for } 1 / 2 \leq x \leq 1 \\
0 & \text { elsewhere }\end{cases} \\
& \tilde{s}_{1}(x)= \begin{cases}0 & \text { for } 0 \leq x<1 / 2 \\
1 & \text { for } 1 / 2 \leq x \leq 1 \\
0 & \text { elsewhere }\end{cases}
\end{aligned}
$$

the MSK modulation scheme can be represented by

$$
\begin{aligned}
& \tilde{s}_{0}(x)= \begin{cases}\frac{1}{\sqrt{2}} & \text { for } 0 \leq x \leq 1 \\
0 & \text { elsewhere }\end{cases} \\
& \tilde{s}_{1}(x)= \begin{cases}\frac{1}{\sqrt{2}} \exp (j \pi x) & \text { for } 0 \leq x \leq 1 \\
0 & \text { elsewhere }\end{cases}
\end{aligned}
$$

and Orthogonal FSK modulation is defined by

$$
\begin{aligned}
& \tilde{s}_{0}(x)= \begin{cases}\frac{1}{\sqrt{2}} & \text { for } 0 \leq x \leq 1 \\
0 & \text { elsewhere }\end{cases} \\
& \tilde{s}_{1}(x)= \begin{cases}\frac{1}{\sqrt{2}} \exp (j 2 \pi x) & \text { for } 0 \leq x \leq 1 \\
0 & \text { elsewhere }\end{cases}
\end{aligned}
$$

All three modulation schemes have the same average energy. According to our observa- 
tions, we have that $N=4$ is large enough for approximating well the fading autocorrelation functions for these cases. Moreover, $L=16$ for Time-Orthogonal modulation and $L=64$ for MSK as well as Orthogonal FSK are large enough to achieve good performance. Thus, in this paper, we use these parameter settings for simulations. Unless explicitly stated, we use the Jakes' model for performance analysis and simulations.

Figure 3 illustrates the computed and simulated BER for Time-Orthogonal modulation with different values of $K$ and $f_{d T}$. We see that increasing $K$ can improve performance. For the lower Doppler $f_{d T}=0.1$, increasing $K$ beyond 4 does not improve performance for SNR less than $50 \mathrm{~dB}$. For larger Doppler $f_{d T}=0.25$, using more than four basis functions can slightly improve performance for SNR $>40 \mathrm{~dB}$. In [13], the authors propose a receiver front-end using specific basis functions to discretize the received continuous time signal, which is simple to implement. In order to show that this receiver has a close to optimal performance, the authors also provide the optimal performance for $f_{d T}=0.1$ as reference. Comparing Figure 3 with [13], we see that our receiver can achieve optimal performance for $K=2,4$ and 6 . To reduce the overall complexity of our scheme we use the FHT algorithm, whose computational complexity is $O(N)$ with $N$ the number of input elements [17] in our receiver front-end.

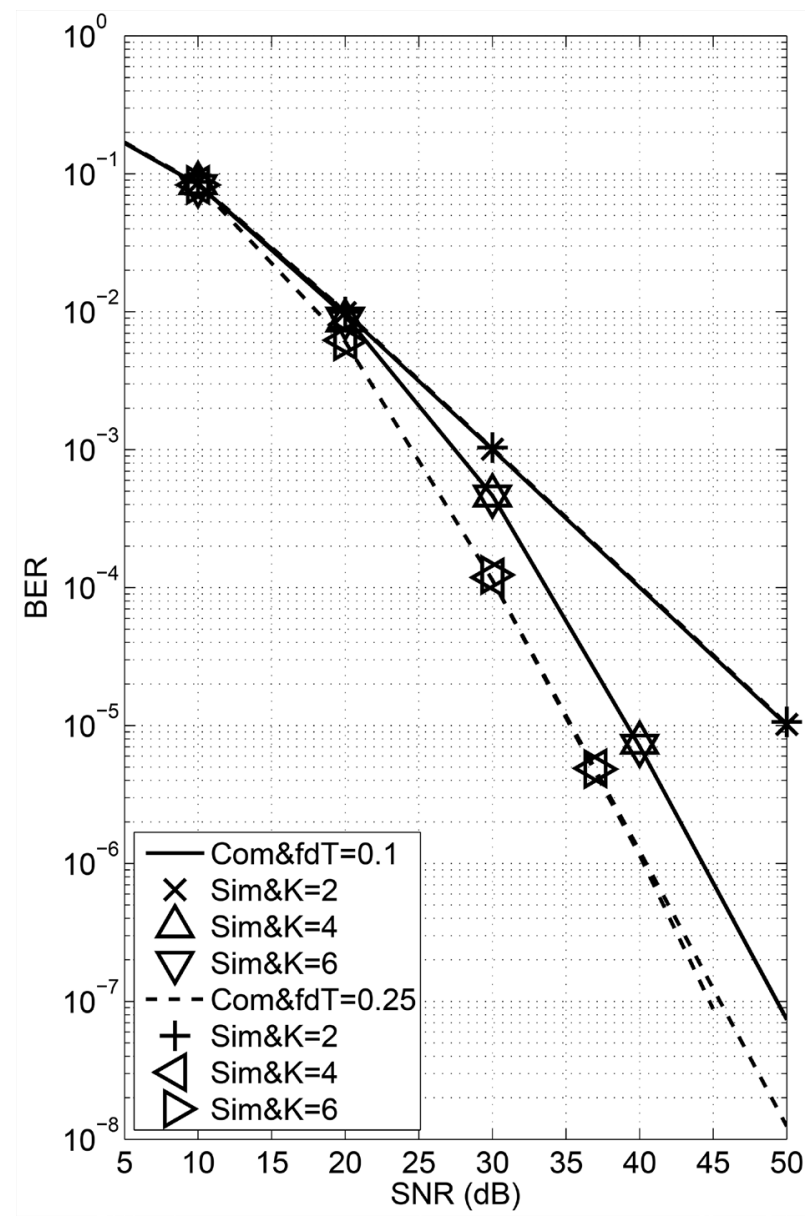

Figure 3. Time-orthogonal modulation, $N=4$ and $L=16$. 
We can find analytically the diversity order that can be obtained with such a Time Orthogonal scheme by using Proposition 2 of [22]. Essentially the result of this proposition is

$$
\lim _{S N R \rightarrow \infty} P_{p}=\text { const. } \cdot S N R^{-K_{q}}
$$

where for $S N R \rightarrow \infty$ the parameter $K_{q}$ is the sum of all positive eigenvalues $\mu_{i}^{0.1}$ of $\mathbf{R}_{0.1}$, that has one additional eigenvalue at -1 with multiplicity $K_{q}$. Figure 4 and Figure 5 present the magnitude of these eigenvalues on a log scale for Time-Orthogonal modulation with $K=4$ and $f_{d T}=0.1$. Figure 4 shows that two positive eigenvalues increase linearly with SNR, and Figure 5 shows that two negative eigenvalues decrease with SNR, converging to -1 . In our case, we have two distinct and positive eigenvalues of multiplicity $q_{l}=1 \quad(l=1,2)$ satisfying $K_{q}=\sum_{l=1}^{2} q_{l}=2$, and a negative eigenvalue of -1 at $S N R \rightarrow \infty$ with multiplicity 2 . Hence, this scheme provides an asymptotic diversity order of two which correlates well with our results of Figure 3 for $K=4,6$.

Figure 6 illustrates the calculated and simulated BER for MSK modulation with different values of $K$ and $f_{d T}$. For $f_{d T}=0.1$, we see that using two basis functions leads to a high error floor, and increasing $K$ to 4 can improve performance and remove the

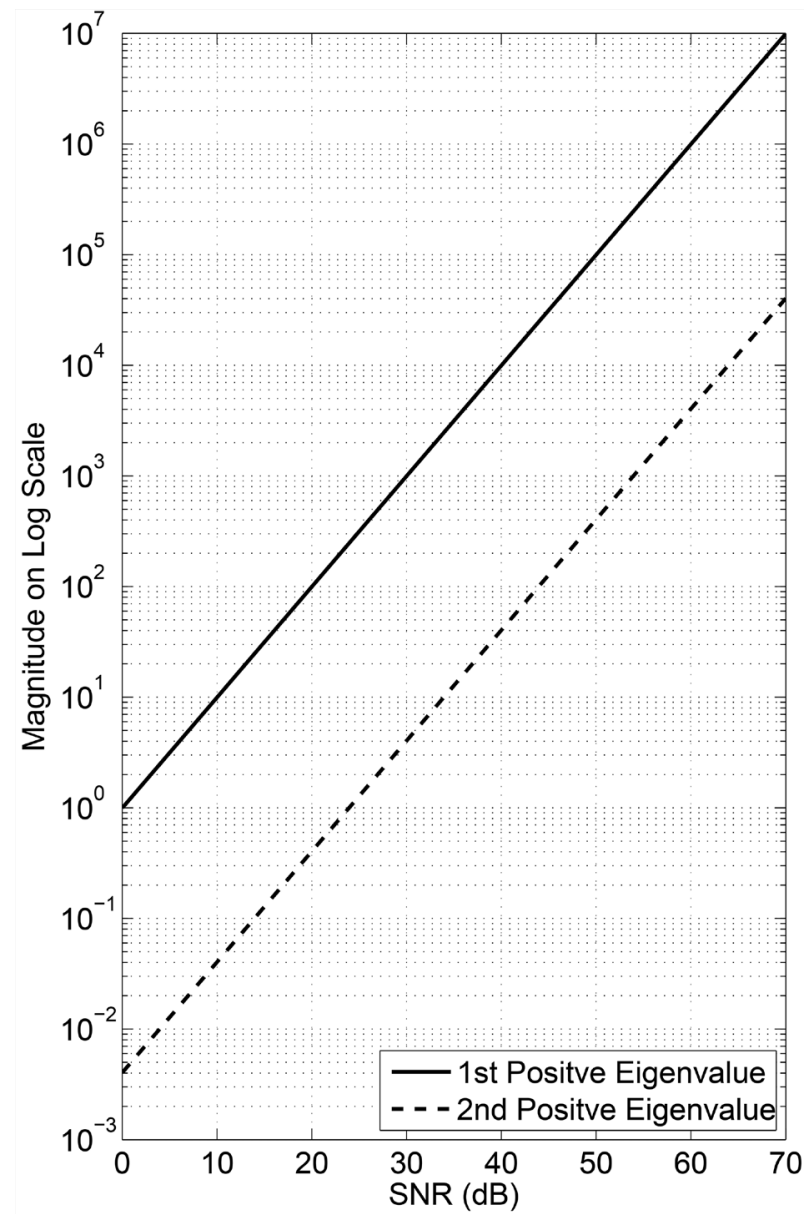

Figure 4. Positive eigenvalues, $N=4, K=4, L=16$ and $f_{d T}=0.1$. 


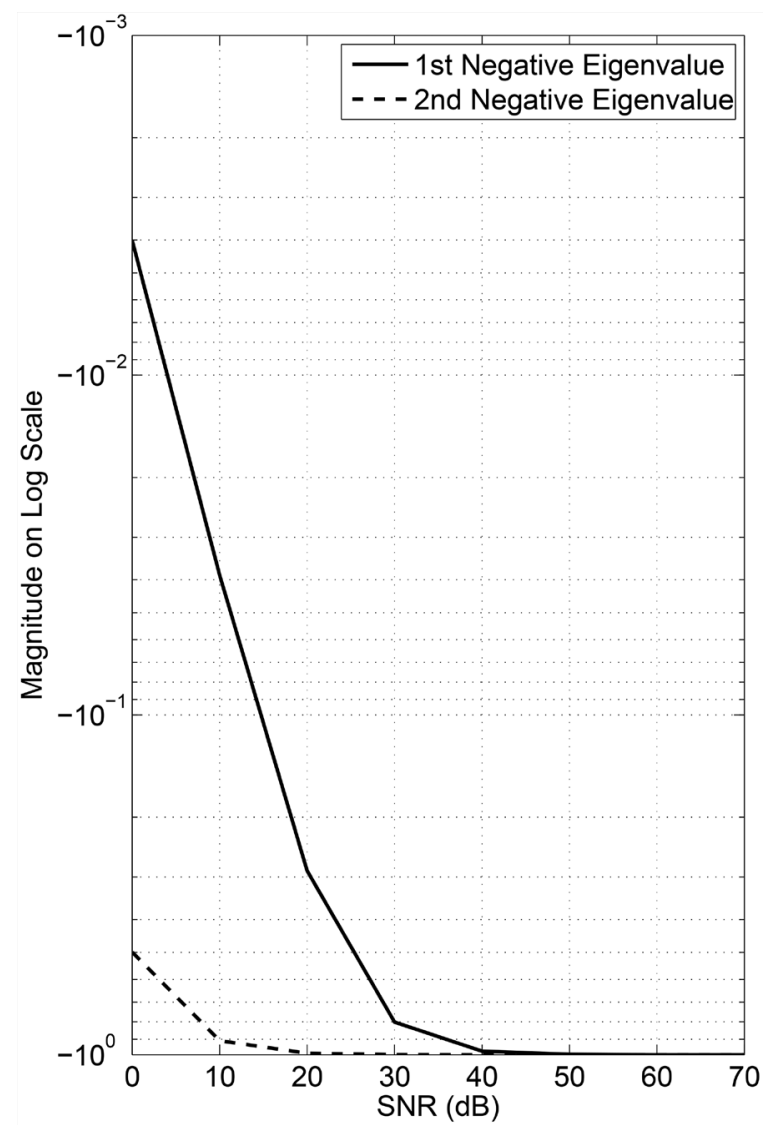

Figure 5. Negative eigenvalues, $N=4, K=4, L=16$ and $f_{d T}=0.1$.

error floor. Using $K=6$ can slightly improve performance further for SNR $>60 \mathrm{~dB}$. For $f_{d T}=0.25$, it is seen that using $K=2$ yields a higher error floor compared to the $f_{d T}=0.1$ case, and increasing $K$ to 4 can lower the error floor by three orders of magnitude. Using six basis functions can further improve performance and remove the error floor. We see that increasing the normalized Doppler spread degrades performance in this case. In [12], the authors present single and double-filter receivers designed for linearly and quadratically time-selective Rayleigh fading channel models. These receivers correspond to our case of two and four basis functions respectively. Performance analysis and simulation results are presented in [12] for MSK modulation. In order to fairly compare our scheme with [12], we use (82), which is the same as ([12], (5.1)), to design the basis functions for the receiver. We generate the fading process using two identical third-order Butterworth lowpass filters as in [12]. Figure 7 illustrates the computed and simulated results for MSK with $f_{d T}=0.1$. Comparing with Figure 4 from [12], we see that the single-filter receiver yields the same performance as our receiver with $K=2$. Comparing with Figure 5 of [12], we see that the double-filter receiver provides the same performance as our receiver with $K=4$ for SNR $<40 \mathrm{~dB}$. For larger SNR, however, our receiver performs better and has an error floor that is more than one order of magnitude lower than the error floor in [12]. Our receiver provides better performance since we approximate the fading process more accurately than in [12]. 


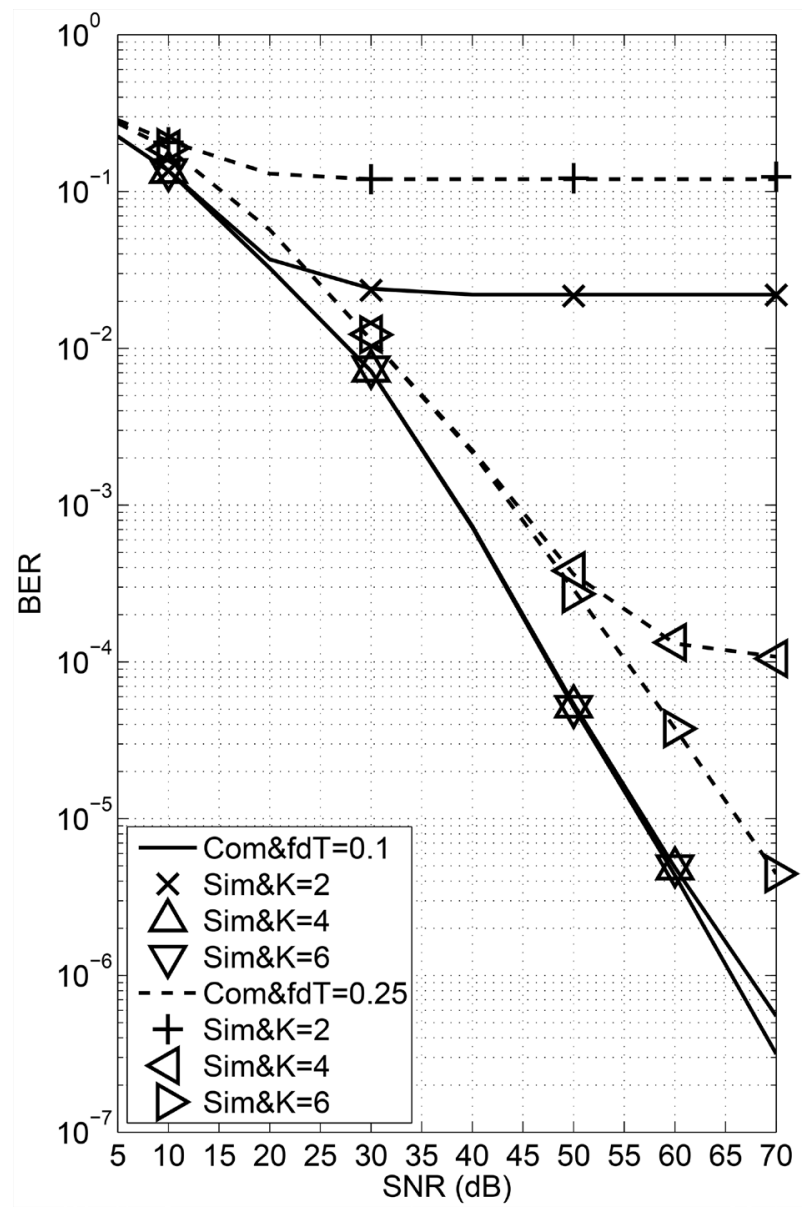

Figure 6. MSK modulation (Jakes), $N=4$ and $L=64$.

From Figure 6 and Figure 7, we see that, for MSK with $f_{d T}=0.1$, the fading spectrum shape affects the performance of our receiver when using $K>2$. With the Jakes' fading spectrum, there is no error floor for $K \geq 4$ and the improvement between $K=$ 4 and $K=6$ is small. With the Butterworth fading spectrum, the performance is worse than with Jakes' fading, and there exists an error floor for $K=4$. When $K=6$, we do not observe an error floor for SNR $\leq 70 \mathrm{~dB}$. With Butterworth fading we see a larger performance improvement when increasing $K$ from 4 to 6 than with Jakes' fading.

Figure 8 illustrates the computed and simulated BER for Orthogonal FSK. We see that for $f_{d T}=0.1$, using $K=2$ leads to a high error floor, while increasing $K$ improves performance and removes the error floor. However, beyond $K=4$, increasing $K$ does not improve performance for SNR less than $50 \mathrm{~dB}$. For $f_{d T}=0.25$ we see that using $K$ $=2$ results in a higher error floor compared to $f_{d T}=0.1$, and increasing $K$ to 4 removes the error floor for SNR below $60 \mathrm{~dB}$. Using $K=6$ can improve performance further for SNR > $35 \mathrm{~dB}$. Orthogonal FSK and Time-Orthogonal modulation are orthogonal signaling schemes with same performance over slow fading channels. However when $f_{d T}$ increases, Orthogonal FSK performs worse than Time-Orthogonal modulation, and better than MSK. 


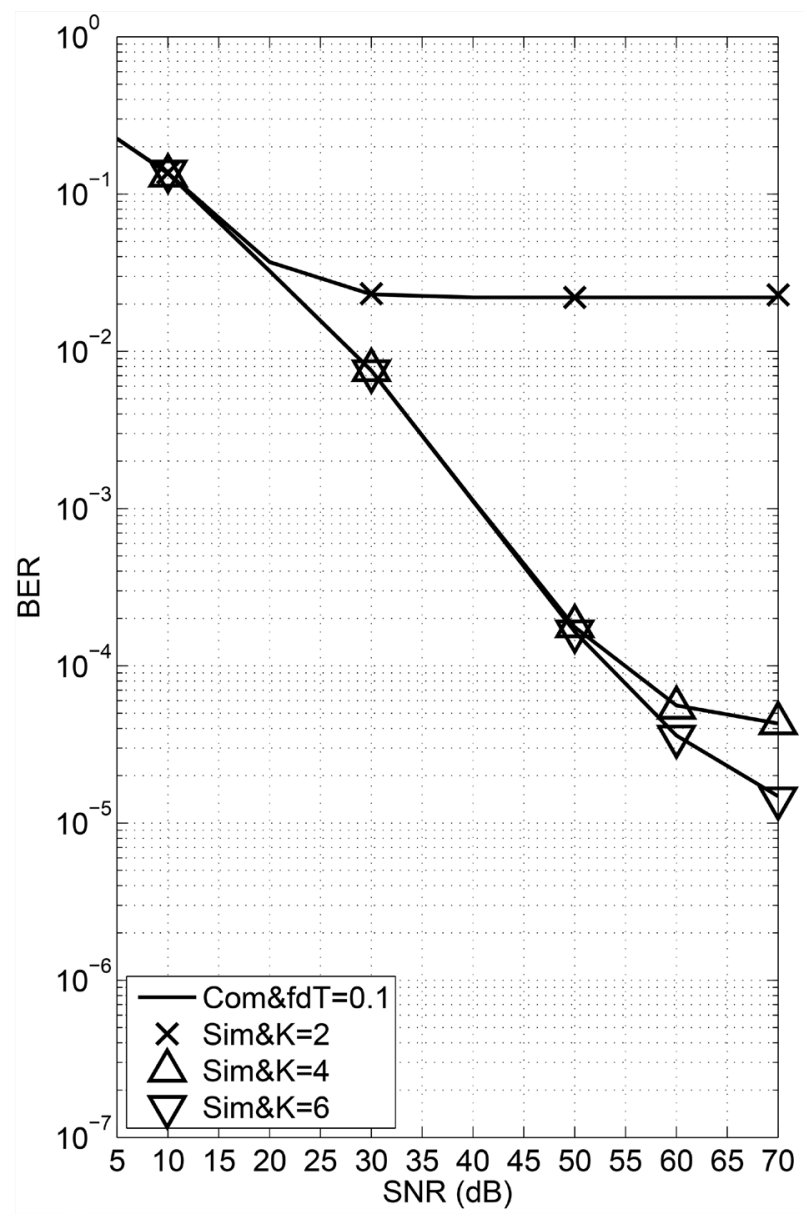

Figure 7. MSK modulation (Butterworth), $N=4, L=64$.

\section{Conclusions}

This paper considers a wavelets based receiver structure for frequency-flat time-varying Rayleigh channels. The receiver consists of a front-end performing discretization of the received continuous time signal, and a MAP detector processing the outputs from the front-end. The fast Haar transform algorithm is used to reduce computational complexity. We present two conditions for achieving optimality over slow-fading channels, and demonstrate that using any orthogonal signaling scheme ensures optimality of our receiver in this case.

Numerical performance analysis and Monte-Carlo simulation results of three binary modulation schemes are presented for fast-fading Rayleigh channels. Among these schemes, Time-Orthogonal modulation performs best, and MSK worst. Increasing $K$, the number of basis function that the receiver uses, improves performance, but when $K>$ 4 the performance is not improved further for Time-Orthogonal modulation and Orthogonal FSK using the Jakes' fading model with $f_{d T}=0.1$. Moreover, not only the Doppler spread but also the fading spectrum shape affects performance. With TimeOrthogonal modulation, our receiver can achieve optimal performance presented in [13] as a reference. For MSK, our receiver using four basis functions can lower the error 


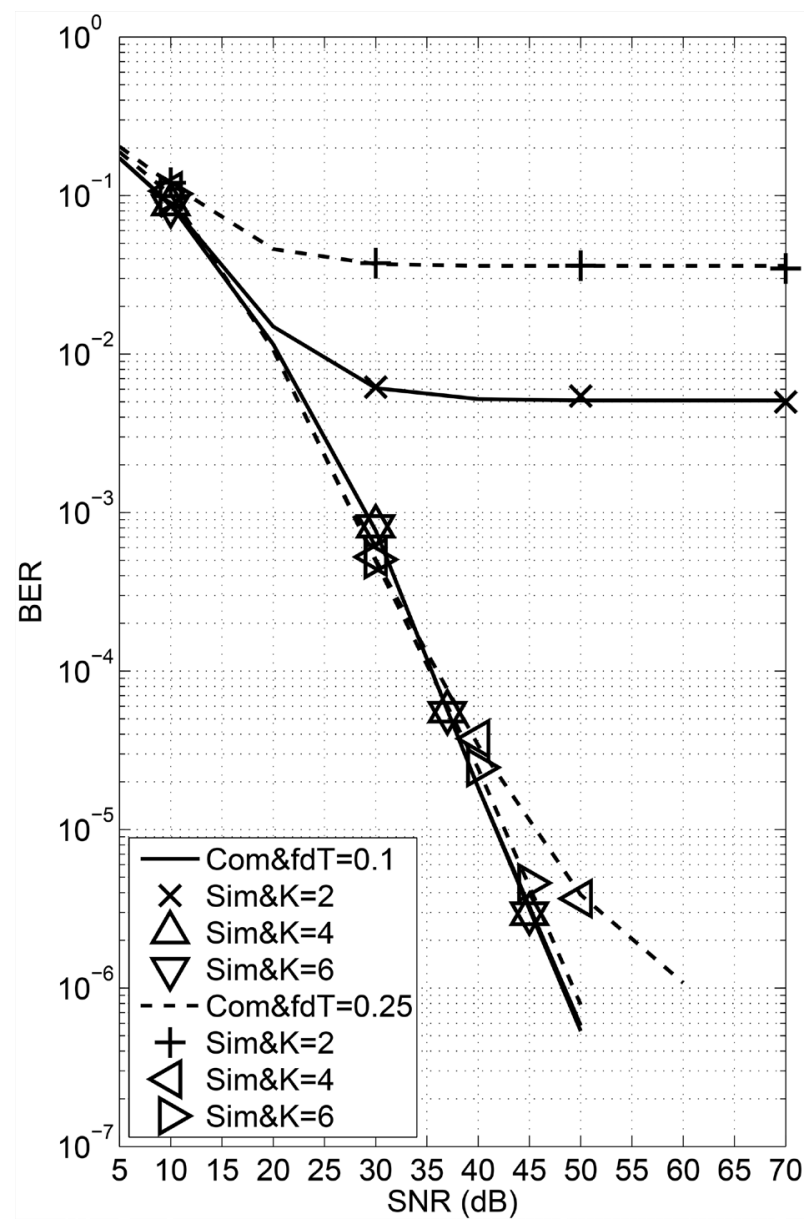

Figure 8. Orthogonal FSK modulation, $N=4$ and $L=64$.

floor by more than one order of magnitude compared to the double-filter receiver of [12]. Orthogonal FSK, which performs the same as Time-Orthogonal modulation over slow fading channels, provides a lower performance over fast time-varying fading channels.

\section{References}

[1] Zhang, K., Zheng, Q., Chatzimisios, P., Xiang, W. and Zhou, Y. (2015) Heterogeneous Vehicular Networking: A Survey on Architecture, Challenges, and Solutions. IEEE Communications Surveys \& Tutorials, 17, 2377-2396. http://dx.doi.org/10.1109/COMST.2015.2440103

[2] Ai, B., Cheng, X., Kürner, T., Zhong, Z.D., Guan, K., He, R.S., Xiong, L., Matolak, D.W., Michelson, D.G. and Briso-Rodriguez, C. (1992) Challenges toward Wireless Communications for High-Speed Railway. IEEE Transactions on Intelligent Transportation Systems, 15, 2143-2158. http://dx.doi.org/10.1109/TITS.2014.2310771

[3] Hlawatsch, F. and Matz, G. (2011) Wireless Communications over Rapidly Time-Varying Channels. Academic Press, London.

[4] Tsai, P., Chang, H., Hsieh, P., Hou, J. and Fan, K. (2010) Baseband Receiver Design for 3GPP Long Term Evolution Downlink OFDMA Systems under Fast-Fading Channels. 2010 
IEEE Asia Pacific Conference on Circuits and Systems (APCCAS), Kuala Lumpur, 6-9 December 2010, 1207-1210. http://dx.doi.org/10.1109/APCCAS.2010.5775016

[5] Rappaport, T. and Fung, V. (1991) Simulation of Bit Error Performance of FSK, BPSK, and $\pi / 4$ DQPSK in Flat Fading Indoor Radio Channels Using a Measurement-Based Channel Model. IEEE Transactions on Vehicular Technology, 40, 731-740.

http://dx.doi.org/10.1109/25.108384

[6] Cavers, J.K. (1992) On the Validity of Slow and Moderate Fading Models for Matched Filter Detection of Rayleigh Fading Signals. Canadian Journal of Electrical and Computer Engineering, 17, 183-189. http://dx.doi.org/10.1109/CJECE.1992.6592505

[7] Bello, P.A. and Nelin, B.D. (1962) The Influence of Fading Spectrum on the Binary Error Probabilities of Incoherent and Differentially Coherent Matched Filter Receivers. IRE Transactions on Communications Systems, 10, 160-168. http://dx.doi.org/10.1109/TCOM.1962.1088654

[8] Cavers, J.K. (1991) An Analysis of Pilot Symbol Assisted Modulation for Rayleigh Fading Channels. IEEE Transactions on Vehicular Technology, 40, 686-693.

http://dx.doi.org/10.1109/25.108378

[9] Fitz, M.P. (1992) Comments on Tone Calibration in Time Varying Fading. IEEE Transactions on Vehicular Technology, 41, 211-213. http://dx.doi.org/10.1109/25.142779

[10] Vietta, G.M. and Taylor, D.P. (1996) Multisampling Receivers for Uncoded and Coded PSK Signal Sequences Transmitted over Rayleigh Frequency-Flat Fading Channels. IEEE Transactions on Communications, 44, 130-133. http://dx.doi.org/10.1109/26.486600

[11] Dam, W.C. and Taylor, D.P. (1994) An Adaptive Maximum Likelihood Receiver for Correlated Rayleigh Flat-Fading Channels. IEEE Transactions on Communications, 42, 26842692. http://dx.doi.org/10.1109/26.317409

[12] Vietta, G.M., Mengali, U. and Taylor, D.P. (1994) Optimal Noncoherent Detection of FSK Signals Transmitted over Linearly Time-Selective Rayleigh Fading Channels. IEEE Transactions on Communications, 45, 1417-1425. http://dx.doi.org/10.1109/26.649762

[13] Hansson, U. and Aulin, T.M. (1999) Aspects on the Single Symbol Signaling on the Frequency Flat Rayleigh Fading Channel. IEEE Transactions on Communications, 47, 874883. http://dx.doi.org/10.1109/26.771344

[14] Visintin, M. (2000) Waveforms for Fast Fading Channels. Electronics Letters, 36, 907-908. http://dx.doi.org/10.1049/el:20000658

[15] Van Trees, H.L. (2001) Detection, Estimation, and Modulation Theory, Part I. Wiley-Interscience, New York.

[16] Shao, X. and Leib, H. (2012) Discretization Methods of Continuous Time Signals over Frequency-Flat Fast-Fading Rayleigh Channels. 25th IEEE Canadian Conference on Electrical \& Computer Engineering (CCECE), Montreal, 29 April-2 May 2012, 1-5. http://dx.doi.org/10.1109/CCECE.2012.6334850

[17] Kaiser, G. (1998) The Fast Haar Transform. Gateway to Wavelet. IEEE Potentials, 17, 3437. http://dx.doi.org/10.1109/45.666645

[18] Proakis, J.G. and Salehi, M. (2008) Digital Communications. 5th Edition, McGraw-Hill, New York.

[19] Phoon, K.K., Huang, S.P. and Quek, S.T. (2002) Implementation of Karhunen-Loeve expansion for Simulation Using a Wavelet-Galerkin Scheme. Probabilistic Engineering Mechanics, 17, 293-303. http://dx.doi.org/10.1016/S0266-8920(02)00013-9

[20] Simon, K.M. and Alouini, M. (2000) Digital Communication over Fading Channels. Wiley-Interscience, New York. http://dx.doi.org/10.1002/0471200697 
[21] Turin, G.L. (1960) The Characteristic Function of Hermitian Quadratic Forms in Complex Normal Variables. Biometrika, 47, 199-201. http://dx.doi.org/10.1093/biomet/47.1-2.199

[22] Brehler, M. and Varansi, M.K. (2001) Asymptotic Error Probability Analysis of Quadratic Receivers in Rayleigh-Fading Channels with Applications to a Unified Analysis of Coherent and Noncoherent Space-Time Receivers. IEEE Transactions on Information Theory, 47, 2383-2399. http://dx.doi.org/10.1109/18.945253

[23] Evgrafov, M. (1966) Analytic Functions. Saunders Mathematics Books, Philadelphia.

[24] Jakes Jr., W.C. (1974) Microwave Mobile Communications. Wiley, New York.

[25] Alimohammad, A., Fard, S.F., Cockburn, B.F. and Schlegel, C. (2002) An Accurate and Compact Rayleigh and Rician Fading Channel Simulator. IEEE Vehicular Technology Conference, Singapore, 11-14 May 2008, 409-413.

[26] Hida, T. and Hitsuda, M. (1993) Gaussian Processes. American Mathematical Society, Providence. 


\section{Appendix}

\section{A. Derivation of the Normalization Factors $a_{k}$ and the Covariance of $\tilde{y}_{k}$}

We derive the factors $a_{k}$ that normalize the basis functions $\tilde{\varphi}_{k}(x)$ to unit energy. We also derive the covariance of $\tilde{y}_{k}$ defined in (8), using the normalized time setting. From (26) we have

$$
a_{k}^{2}=\int_{0}^{1} \frac{1}{\tilde{\lambda}_{k}} \sum_{i=0}^{N M-1} \omega_{i} \tilde{b}_{i}(x) \tilde{\phi}_{i}^{(k)} \frac{1}{\tilde{\lambda}_{k}} \sum_{j=0}^{N M-1} \omega_{j} \tilde{b}_{j}^{*}(x) \tilde{\phi}_{j}^{(k) *} \mathrm{~d} x .
$$

Since $\quad \tilde{b}_{i}(x)=\theta_{n}(x) \tilde{s}_{u}(x) \quad$ with $\quad i=n M+u \quad$ and $\quad \tilde{b}_{j}(x)=\theta_{w}(x) \tilde{s}_{v}(x) \quad$ with $j=w M+v$, we have

$$
\begin{aligned}
& a_{k}^{2}=\frac{1}{\tilde{\lambda}_{k}^{2}} \sum_{i=0}^{N M-1} \omega_{i} \tilde{\phi}_{i}^{(k)} \sum_{j=0}^{N M-1} \omega_{j} \tilde{\phi}_{j}^{(k) *} \int_{0}^{1} \tilde{s}_{u}(x) \theta_{n}(x) \tilde{s}_{v}^{*}(x) \theta_{w}(x) \mathrm{d} x \\
& =\frac{1}{\tilde{\lambda}_{k}^{2}} \sum_{i=0}^{N M-1} \omega_{i} \tilde{\phi}_{i}^{(k)} \sum_{j=0}^{N M-1} \omega_{j} \tilde{\phi}_{j}^{(k) *} \sum_{e=0}^{L-1} \frac{\tilde{d}_{e}^{(n)}}{h_{e}^{1 / 2}} \sum_{f=0}^{L-1} \frac{\tilde{d}_{f}^{(w)}}{h_{f}^{1 / 2}} \int_{0}^{1} \tilde{s}_{u}(x) \psi_{e}(x) \tilde{s}_{v}^{*}(x) \psi_{f}(x) \mathrm{d} x \\
& =\frac{1}{\tilde{\lambda}_{k}^{2}} \sum_{i=0}^{N M-1} \omega_{i} \tilde{\phi}_{i}^{(k)} \sum_{j=0}^{N M-1} \omega_{j} \tilde{\phi}_{j}^{(k) *} \sum_{e=0}^{L-1} \frac{\tilde{d}_{e}^{(n)}}{h_{e}^{1 / 2}} \sum_{f=0}^{L-1} \frac{\tilde{d}_{f}^{(w)}}{h_{f}^{1 / 2}} \sum_{p=0}^{R-1} \psi_{p}^{e} \psi_{p}^{f} \int_{\frac{p}{R}}^{\frac{p+1}{R}} \tilde{s}_{u}(x) \tilde{s}_{v}^{*}(x) \mathrm{d} x
\end{aligned}
$$

where (96) is obtained using (33), and (97) is due to $\psi_{e}(x), \psi_{f}(x)$ being constant over each integration sub-interval with $\psi_{p}^{e}$ and $\psi_{p}^{f}$ defined in (43).

The covariance of $\tilde{y}_{k}$ can be expressed as

$$
\begin{aligned}
& E\left[\tilde{y}_{k} \tilde{y}_{l}^{*} \mid \tilde{s}_{m}(x)\right]=E\left[\int_{0}^{1} \tilde{g}\left(x_{1}\right) \tilde{s}_{m}\left(x_{1}\right) \tilde{\varphi}_{k}^{*}\left(x_{1}\right) \mathrm{d} x_{1} \int_{0}^{1} \tilde{g}^{*}\left(x_{2}\right) \tilde{s}_{m}^{*}\left(x_{2}\right) \tilde{\varphi}_{l}\left(x_{2}\right) \mathrm{d} x_{2}\right] \\
& =\sum_{i=0}^{N M-1} \frac{\omega_{i} \tilde{\phi}_{i}^{(k) *}}{a_{k} \tilde{\lambda}_{k}} \sum_{j=0}^{N M-1} \frac{\omega_{j} \tilde{\phi}_{j}^{(l)}}{a_{l} \tilde{\lambda}_{l}} E\left[\int_{0}^{1} \tilde{g}\left(x_{1}\right) \tilde{s}_{m}\left(x_{1}\right) \tilde{b}_{i}^{*}\left(x_{1}\right) \mathrm{d} x_{1} \int_{0}^{1} \tilde{g}^{*}\left(x_{2}\right) \tilde{s}_{m}^{*}\left(x_{2}\right) \tilde{b}_{j}\left(x_{2}\right) \mathrm{d} x_{2}\right] .
\end{aligned}
$$

Due to $\tilde{b}_{i}(x)=\theta_{n}(x) \tilde{s}_{u}(x) \quad i=n M+u$ and $\tilde{b}_{j}(x)=\theta_{w}(x) \tilde{s}_{v}(x) \quad j=w M+v$, we have

$$
\begin{aligned}
E & {\left[\tilde{y}_{k} \tilde{y}_{l}^{*} \mid \tilde{s}_{m}(x)\right]=\sum_{i=0}^{N M-1} \frac{\omega_{i} \tilde{\phi}_{i}^{(k) *}}{a_{k} \tilde{\lambda}_{k}} \sum_{j=0}^{N M-1} \frac{\omega_{j} \tilde{\phi}_{j}^{(l)}}{a_{l} \tilde{\lambda}_{l}} E\left[\int_{0}^{1} \tilde{g}\left(x_{1}\right) \tilde{s}_{m}\left(x_{1}\right) \tilde{s}_{u}^{*}\left(x_{1}\right) \theta_{n}\left(x_{1}\right) \mathrm{d} x_{1}\right.} \\
& \left.\cdot \int_{0}^{1} \tilde{g}^{*}\left(x_{2}\right) \tilde{s}_{m}^{*}\left(x_{2}\right) \tilde{s}_{v}\left(x_{2}\right) \theta_{w}\left(x_{2}\right) \mathrm{d} x_{2}\right] \\
= & \sum_{i=0}^{N M-1} \frac{\omega_{i} \tilde{\phi}_{i}^{(k) *}}{a_{k} \tilde{\lambda}_{k}} \sum_{j=0}^{N M-1} \frac{\omega_{j} \tilde{\phi}_{j}^{(l)}}{a_{l} \tilde{\lambda}_{l}} \sum_{e=0}^{L-1} \frac{\tilde{d}_{e}^{(n)}}{h_{e}^{1 / 2}} \sum_{f=0}^{L-1} \frac{\tilde{d}_{f}^{(w)}}{h_{f}^{1 / 2}} E\left[\int_{0}^{1} \tilde{g}\left(x_{1}\right) \tilde{s}_{m}\left(x_{1}\right) \tilde{s}_{u}^{*}\left(x_{1}\right) \psi_{e}\left(x_{1}\right) \mathrm{d} x_{1}\right. \\
& \left.\cdot \int_{0}^{1} \tilde{g}^{*}\left(x_{2}\right) \tilde{s}_{m}^{*}\left(x_{2}\right) \tilde{s}_{v}\left(x_{2}\right) \psi_{f}\left(x_{2}\right) \mathrm{d} x_{2}\right] \\
= & \sum_{i=0}^{N M-1} \frac{\omega_{i} \tilde{\phi}_{i}^{(k) *}}{a_{k} \tilde{\lambda}_{k}} \sum_{j=0}^{N M-1} \frac{\omega_{j} \tilde{\phi}_{j}^{(l)}}{a_{l} \tilde{\lambda}_{l}} \sum_{e=0}^{L-1} \frac{\tilde{d}_{e}^{(n)}}{h_{e}^{1 / 2}} \sum_{f=0}^{L-1} \frac{\tilde{d}_{f}^{(w)}}{h_{f}^{1 / 2}} \sum_{p=0}^{R-1} \psi_{p}^{e} \sum_{q=0}^{R-1} \psi_{q}^{f} \int_{\frac{p}{R}}^{\frac{p+1}{R}} \frac{\int_{q}}{R} \\
& \cdot E\left[\tilde{g}\left(x_{1}\right) \tilde{g}^{*}\left(x_{2}\right)\right] \tilde{s}_{m}\left(x_{1}\right) \tilde{s}_{u}^{*}\left(x_{1}\right) \tilde{s}_{m}^{*}\left(x_{2}\right) \tilde{s}_{v}\left(x_{2}\right) \mathrm{d} x_{1} \mathrm{~d} x_{2}
\end{aligned}
$$

where (101) is obtained using (33), and (102) is due to $\psi_{e}(x), \psi_{f}(x)$ being constant 
over each integration sub-interval, with $\psi_{p}^{e}$ and $\psi_{q}^{f}$ defined in (43).

\section{B. Conditions 1 and 2}

We show that satisfying (71) in Section 3.3 is sufficient for Conditions 1 and 2 to hold. Assume that $R_{\tilde{g}}\left(x_{1}, x_{2}\right)=\rho_{g}^{2}$. Because of (71), from (98), we obtain

$$
\begin{aligned}
& E\left[\tilde{y}_{k} \tilde{y}_{l}^{*} \mid \tilde{s}_{m}(x)\right]=E\left[\int_{0}^{1} \tilde{g}\left(x_{1}\right) \tilde{s}_{m}\left(x_{1}\right) \tilde{\varphi}_{k}^{*}\left(x_{1}\right) \mathrm{d} x_{1} \int_{0}^{1} \tilde{g}^{*}\left(x_{2}\right) \tilde{s}_{m}^{*}\left(x_{2}\right) \tilde{\varphi}_{l}\left(x_{2}\right) \mathrm{d} x_{2}\right] \\
& =\rho_{g}^{2} \int_{0}^{1} \tilde{s}_{m}\left(x_{1}\right) \tilde{\varphi}_{k}^{*}\left(x_{1}\right) d x_{1} \int_{0}^{1} \tilde{s}_{m}^{*}\left(x_{2}\right) \tilde{\varphi}_{l}\left(x_{2}\right) \mathrm{d} x_{2}=\rho_{g}^{2} \tilde{E}_{m} \delta_{m k} \delta_{m l}
\end{aligned}
$$

In this case, from (48) the covariance matrix can be expressed as

$$
\tilde{\mathbf{C}}_{m}=\left[\begin{array}{cccc}
0 & 0 & \cdots & 0 \\
0 & \rho_{g}^{2} \tilde{E}_{m} & \cdots & 0 \\
\vdots & \vdots & \ddots & \vdots \\
0 & 0 & \cdots & 0
\end{array}\right]+\frac{N_{0}}{T} \mathbf{I}_{K}=\left[\begin{array}{cccc}
N_{0} / T & 0 & \cdots & 0 \\
0 & \rho_{g}^{2} \tilde{E}_{m}+\left(N_{0} / T\right) & \cdots & 0 \\
\vdots & \vdots & \ddots & \vdots \\
0 & 0 & \cdots & N_{0} / T
\end{array}\right]
$$

and hence, using (67),

$$
\ln \left|\tilde{\mathbf{C}}_{m}\right|=\ln \left(\left(\frac{\rho_{g}^{2} \tilde{E}_{m}}{N_{0} / T}+1\right)\left(N_{0} / T\right)^{K}\right)=\ln \left(S N R_{m}+1\right)+K \ln \left(N_{0} / T\right)
$$

where $K \ln \left(N_{0} / T\right)$ is a constant. Thus, Condition 1 holds.

The inverse of $\tilde{\mathbf{C}}_{m}$ is

$$
\begin{aligned}
& \tilde{\mathbf{C}}_{m}^{-1}=\left[\begin{array}{cccc}
\frac{1}{N_{0} / T} & 0 & \cdots & 0 \\
0 & \frac{N_{0} / T}{\left[\rho_{g}^{2} \tilde{E}_{m}+\left(N_{0} / T\right)\right]\left(N_{0} / T\right)} & \cdots & 0 \\
\vdots & \vdots & \ddots & \vdots \\
0 & 0 & \cdots & \frac{1}{N_{0} / T}
\end{array}\right] \\
& =\left[\begin{array}{cccc}
\frac{1}{N_{0} / T} & 0 & \cdots & 0 \\
0 & \frac{\left(N_{0} / T\right)+\rho_{g}^{2} \tilde{E}_{m}-\rho_{g}^{2} \tilde{E}_{m}}{\left[\rho_{g}^{2} \tilde{E}_{m}+\left(N_{0} / T\right)\right]\left(N_{0} / T\right)} & \cdots & 0 \\
\vdots & \vdots & \ddots & \vdots \\
0 & 0 & \cdots & \frac{1}{N_{0} / T}
\end{array}\right] \\
& =\left[\begin{array}{cccc}
0 & 0 & \cdots & 0 \\
0 & -\frac{\rho_{g}^{2} \tilde{E}_{m}}{\left[\rho_{g}^{2} \tilde{E}_{m}+\left(N_{0} / T\right)\right]\left(N_{0} / T\right)} & \cdots & 0 \\
\vdots & \vdots & \ddots & \vdots \\
0 & 0 & \cdots & 0
\end{array}\right]+\frac{1}{N_{0} / T} \mathbf{I}_{K}=\mathbf{B}_{m}^{\prime}+\mathbf{M}_{c}^{\prime}
\end{aligned}
$$


where $\mathbf{M}_{c}^{\prime}=\frac{1}{N_{0} / T} \mathbf{I}_{K}$ is a constant matrix. Therefore, due to (68) and (109), we have

$$
\begin{aligned}
& \mathbf{L}_{c}^{\prime H} \tilde{\mathbf{C}}_{m}^{-1} \mathbf{L}_{c}^{\prime}=\mathbf{L}_{c}^{\prime H} \mathbf{B}_{m}^{\prime} \mathbf{L}_{c}^{\prime}+\mathbf{L}_{c}^{\prime H} \mathbf{M}_{c}^{\prime} \mathbf{L}_{c}^{\prime} \\
& =\mathbf{L}_{c}^{\prime H}\left[\begin{array}{cccc}
0 & 0 & \cdots & 0 \\
0 & -\frac{\rho_{g}^{2} \tilde{E}_{m}}{\left[\rho_{g}^{2} \tilde{E}_{m}+\left(N_{0} / T\right)\right]\left(N_{0} / T\right)} & \cdots & 0 \\
\vdots & \vdots & \ddots & \vdots \\
0 & 0 & \cdots & 0
\end{array}\right] \mathbf{L}_{c}^{\prime}+\mathbf{L}_{c}^{\prime H} \mathbf{M}_{c}^{\prime} \mathbf{L}_{c}^{\prime}=\mathbf{B}_{m}+\mathbf{M}_{c}
\end{aligned}
$$

where $\mathbf{M}_{c}=\mathbf{L}_{c}^{\prime H} \mathbf{M}_{c}^{\prime} \mathbf{L}_{c}^{\prime}$ is a constant matrix because $\mathbf{M}_{c}^{\prime}$ is a constant matrix. Because of (60), $\mathbf{B}_{m}$ can be expressed as

$$
\mathbf{B}_{m}=\mathbf{L}_{c}^{\prime H} \mathbf{B}_{m}^{\prime} \mathbf{L}_{c}^{\prime}
$$

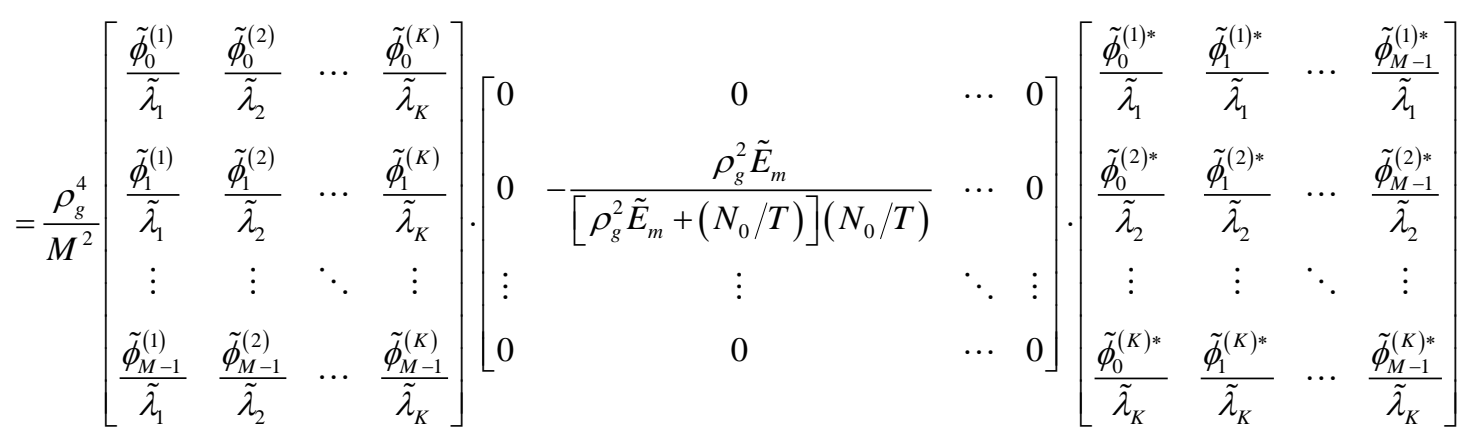

$=\frac{\rho_{g}^{4}}{M^{2}}\left[\begin{array}{cccc}0 & -\frac{\rho_{g}^{2} \tilde{E}_{m} \tilde{\lambda}_{m}^{-1} \tilde{\phi}_{0}^{(m)}}{\left[\rho_{g}^{2} \tilde{E}_{m}+\left(N_{0} / T\right)\right]\left(N_{0} / T\right)} & \cdots & 0 \\ 0 & -\frac{\rho_{g}^{2} \tilde{E}_{m} \tilde{\lambda}_{m}^{-1} \tilde{\phi}_{1}^{(m)}}{\left[\rho_{g}^{2} \tilde{E}_{m}+\left(N_{0} / T\right)\right]\left(N_{0} / T\right)} & \cdots & 0 \\ \vdots & \ddots & \vdots \\ 0 & -\frac{\rho_{g}^{2} \tilde{E}_{m} \tilde{\lambda}_{m}^{-1} \tilde{\phi}_{M-1}^{(m)}}{\left[\rho_{g}^{2} \tilde{E}_{m}+\left(N_{0} / T\right)\right]\left(N_{0} / T\right)} & \cdots & 0\end{array}\right] \cdot\left[\begin{array}{cccc}\frac{\tilde{\phi}_{0}^{(1) *}}{\tilde{\lambda}_{1}} & \frac{\tilde{\phi}_{1}^{(1) *}}{\tilde{\lambda}_{1}} & \cdots & \frac{\tilde{\phi}_{M-1}^{(1) *}}{\tilde{\lambda}_{1}} \\ \frac{\tilde{\phi}_{0}^{(2) *}}{\tilde{\lambda}_{2}} & \frac{\tilde{\phi}_{1}^{(2) *}}{\tilde{\lambda}_{2}} & \cdots & \frac{\tilde{\phi}_{M-1}^{(2) *}}{\tilde{\lambda}_{2}} \\ \vdots & \vdots & \ddots & \vdots \\ \frac{\tilde{\phi}_{0}^{(K) *}}{\tilde{\lambda}_{K}} & \frac{\tilde{\phi}_{1}^{(K) *}}{\tilde{\lambda}_{K}} & \cdots & \frac{\tilde{\phi}_{M-1}^{(K) *}}{\tilde{\lambda}_{K}}\end{array}\right]$

$$
=\frac{\rho_{g}^{4}}{M^{2}}\left[\begin{array}{cccc}
-\frac{\rho_{g}^{2} \tilde{E}_{m} \tilde{\lambda}_{m}^{-2} \tilde{\phi}_{0}^{(m)} \tilde{\phi}_{0}^{(m) *}}{\left[\rho_{g}^{2} \tilde{E}_{m}+\left(N_{0} / T\right)\right]\left(N_{0} / T\right)} & -\frac{\rho_{g}^{2} \tilde{E}_{m} \tilde{\lambda}_{m}^{-2} \tilde{\phi}_{0}^{(m)} \tilde{\phi}_{1}^{(m) *}}{\left[\rho_{g}^{2} \tilde{E}_{m}+\left(N_{0} / T\right)\right]\left(N_{0} / T\right)} & \cdots & -\frac{\rho_{g}^{2} \tilde{E}_{m} \tilde{\lambda}_{m}^{-2} \tilde{\phi}_{0}^{(m)} \tilde{\phi}_{M-1}^{(m) *}}{\left[\rho_{g}^{2} \tilde{E}_{m}+\left(N_{0} / T\right)\right]\left(N_{0} / T\right)} \\
-\frac{\rho_{g}^{2} \tilde{E}_{m} \tilde{\lambda}_{m}^{-2} \tilde{\phi}_{1}^{(m)} \tilde{\phi}_{0}^{(m) *}}{\left[\rho_{g}^{2} \tilde{E}_{m}+\left(N_{0} / T\right)\right]\left(N_{0} / T\right)} & -\frac{\rho_{g}^{2} \tilde{E}_{m} \tilde{\lambda}_{m}^{-2} \tilde{\phi}_{1}^{(m)} \tilde{\phi}_{1}^{(m) *}}{\left[\rho_{g}^{2} \tilde{E}_{m}+\left(N_{0} / T\right)\right]\left(N_{0} / T\right)} & \cdots & -\frac{\rho_{g}^{2} \tilde{E}_{m} \tilde{\lambda}_{m}^{-2} \tilde{\phi}_{1}^{(m)} \tilde{\phi}_{M-1}^{(m) *}}{\left[\rho_{g}^{2} \tilde{E}_{m}+\left(N_{0} / T\right)\right]\left(N_{0} / T\right)} \\
\vdots & \ddots & \vdots \\
-\frac{\rho_{g}^{2} \tilde{E}_{m} \tilde{\lambda}_{m}^{-2} \tilde{\phi}_{M-1}^{(m)} \tilde{\phi}_{0}^{(m) *}}{\left[\rho_{g}^{2} \tilde{E}_{m}+\left(N_{0} / T\right)\right]\left(N_{0} / T\right)} & -\frac{\rho_{g}^{2} \tilde{E}_{m} \tilde{\lambda}_{m}^{-2} \tilde{\phi}_{M-1}^{(m)} \tilde{\phi}_{1}^{(m) *}}{\left[\rho_{g}^{2} \tilde{E}_{m}+\left(N_{0} / T\right)\right]\left(N_{0} / T\right)} & \cdots & -\frac{\rho_{g}^{2} \tilde{E}_{m} \tilde{\lambda}_{m}^{-2} \tilde{\phi}_{M-1}^{(m)} \tilde{\phi}_{M-1}^{(m) *}}{\left[\rho_{g}^{2} \tilde{E}_{m}+\left(N_{0} / T\right)\right]\left(N_{0} / T\right)}
\end{array}\right] .
$$

Due to (67) and (71), (115) becomes 


$$
\begin{aligned}
& \mathbf{B}_{m}=\left[\begin{array}{cccc}
0 & 0 & \cdots & 0 \\
0 & -\frac{\rho_{g}^{4} \tilde{E}_{m}}{M^{2} \tilde{\lambda}_{m}^{2}} \cdot \frac{\rho_{g}^{2} \tilde{E}_{m}}{\left[\rho_{g}^{2} \tilde{E}_{m}+\left(N_{0} / T\right)\right]\left(N_{0} / T\right)} & \cdots & 0 \\
\vdots & \vdots & \ddots & \vdots \\
0 & 0 & \cdots & 0
\end{array}\right] \\
& =\left[\begin{array}{cccc}
0 & 0 & \cdots & 0 \\
0 & -\frac{\rho_{g}^{4} \tilde{E}_{m}}{M^{2} \tilde{\lambda}_{m}^{2}\left(N_{0} / T\right)} \cdot \frac{S N R_{m}}{S N R_{m}+1} & \cdots & 0 \\
\vdots & \vdots & \ddots & \vdots \\
0 & 0 & \cdots & 0
\end{array}\right]
\end{aligned}
$$

where the non-zero component of $\mathbf{B}$ can be put in the form

$$
b_{m, m}=-\frac{\rho_{g}^{4} \tilde{E}_{m}}{M^{2} \tilde{\lambda}_{m}^{2}\left(N_{0} / T\right)} \cdot \frac{S N R_{m}}{S N R_{m}+1}=-\frac{\rho_{g}^{4} E_{m}}{M^{2} \tilde{\lambda}_{m}^{2} N_{0}} \cdot \frac{S N R_{m}}{S N R_{m}+1}=-\frac{\rho_{g}^{4} E_{m}^{2}}{M^{2} \tilde{\lambda}_{m}^{2} E_{m} N_{0}} \cdot \frac{S N R_{m}}{S N R_{m}+1} .
$$

When $\frac{\tilde{E}_{m}}{\tilde{\lambda}_{m}}$ is constant we have $b_{m, m}=\frac{c_{S}}{E_{m} N_{0}} \cdot \frac{S N R_{m}}{S N R_{m}+1}$ where $c_{S}$ is a constant.

Therefore, Condition 2 also holds.

Submit or recommend next manuscript to SCIRP and we will provide best service for you:

Accepting pre-submission inquiries through Email, Facebook, LinkedIn, Twitter, etc.

A wide selection of journals (inclusive of 9 subjects, more than 200 journals)

Providing 24-hour high-quality service

User-friendly online submission system

Fair and swift peer-review system

Efficient typesetting and proofreading procedure

Display of the result of downloads and visits, as well as the number of cited articles

Maximum dissemination of your research work

Submit your manuscript at: http://papersubmission.scirp.org/

Orcontactijcns@scirp.org 